\title{
Single Cell Proteomics Profiling Revealing that Embryo Secreted TNF-a play Critical Role during Embryo Implantation to Endometrium
}

\section{Lv Jiao ( 253308380@qq.com )}

Sichuan University West China Second University Hospital https://orcid.org/0000-0002-1315-1807

\section{Shan Xudong}

Chengdu University of Traditional Chinese Medicine

\section{Yang Haoxuan}

Sichuan University West China Second University Hospital

\section{Wen Yuting}

Sichuan University West China Second University Hospital

\section{Zhang Xueguang}

Sichuan University West China Second University Hospital

\section{Chen Hanxiao}

Sichuan University West China Second University Hospital

\section{Li Hengli}

Chengdu University of Traditional Chinese Medicine

\section{Tian Dongmei}

Chengdu University of Traditional Chinese Medicine

\section{Wang Chi Chiu}

The Chinese University of Hong Kong

\section{Zhang Ruizhe}

The Chinese University of Hong Kong

\section{Li Tin Chiu}

The Chinese University of Hong Kong

\section{Zhang Xiaohu}

Sichuan University West China Second University Hospital

\section{Zhao Xiaomiao}

Sun Yat-Sen Memorial Hospital

\section{Lu Yao}

Chinese Academy of Sciences

\section{Qin Lang}

Sichuan University West China Second University Hospital

\section{Zhu Minghui}


Chengdu University of Traditional Chinese Medicine

Xu Wenming

Sichuan University West China Second University Hospital

Research

Keywords: Culture medium, embryo quality, TNF-a, IVF outcome

Posted Date: December 10th, 2020

DOl: https://doi.org/10.21203/rs.3.rs-122459/v1

License: (c) (1) This work is licensed under a Creative Commons Attribution 4.0 International License. Read Full License 


\section{Abstract}

Problem: Although it has long been known that endometrium secreted cytokines play critical role during embryo implantation, whether cytokines secreted from embryo is relevant to embryo quality and is actively involved in embryo attachment remain unclear.

Method of study: The concentration of cytokines in embryo culture medium were tested by a new developed high-sensitive single cell proteomic platform, compared with embryo quality and clinical outcome. The effect of TNF-a on embryo and endometrium Ishikawa cell was investigated using immunofluorescence staining, CCK- 8 assay, TUNEL staining, and RT-qPCR reaction.

Results: Of the 10 cytokines measured, only TNF-a concentration is significantly higher in group of embryo implantation failure. Immunofluorescence staining showed that the expression of TNF-a was unevenly distributed in blastocysts, and the expression level was significantly correlated with blastocysts inner cell mass (ICM) quality score. Adding TNF-a caused significant increase of apoptotic cells, which could be inhibited by TNF-a receptor blocker entanecept (ETA). Gene profiling showed that adding TNF-a lead to increased expression of TNFR1 and apoptosis related genes, as well as ion channel genes, including CFTR, ENaCA, AQP3 and CRISP2, and the increase can be inhibited by ETA.

Conclusion: In conclusion, our result showed that higher TNF-a level is associated with implantation failure through activation of TNF-a receptor, and TNF-a maybe an independent predictor for pre-transfer assessment of the embryo development potential in IVF patients.

\section{Introduction}

Embryo implantation is a highly orchestrated process, which is related to the close interaction of embryo and endometrium. During this process, the invasion of embryo is accompanied with cytokine release, free DNA and secretion of metabolites, ${ }^{1,2}$ which constitute the major molecular determinants critical for embryo invasion. Cytokine release, specially such as TNF-a. Interleukin release secreted from the endometrium, has long been shown be involved in the process since embryo invasion is a controlled inflammation process highly regulated by immune factors. ${ }^{3}$ Recent study has shown that embryo can also actively express immune molecules during development and play roles in the interaction with endometrium. Using mice embryo activation model, Wang et al. has found that several cytokines, including TNF-a, is activated when embryo form dominancy to the activated status and play roles in Xchromosome reactivation. ${ }^{4}$ Therefore, dysregulation of these factors would potentially cause the skewed cross-talk of embryo and endometrium, which could lead to implantation failure in clinic.

Implantation failure is, by definition, a fact that the embryo fails to achieve clinical pregnancy after the embryos implanted during IVF process. ${ }^{5}$ Repeated implantation failure is accounted for at least $50 \%$ cases of IVF failure. ${ }^{6}$ The failure of embryo implantation process can be a consequence of uterine, 
embryo and male factors, ${ }^{7}$ therefore it is vital to identify the biomarkers in advance since the early detection of biomarker could beneficial for the decision of the reproduction process.

Like the nature condition, the embryonic factor is also critical for the embryo implantation process during in vitro culture in IVF process. For example, it has been well known that embryo can secret different kinds of protein and nucleotides to pave the way for the successful invasion. ${ }^{8}$ Although cytokines have been long studied and used as biomarker in different diseases condition, including in preterm labor and preeclampsia, ${ }^{9}$ the analysis of the cytokines in embryo culture medium has always been a challenge since it has few volume, low concentration, and it may be secreted with other forms such as exosome and micro-vesicle, therefore it increase the difficulty for quantitative detection. Recent advance in single molecule, microfluidic analysis has improved the detection to more sensitive level. ${ }^{10}$ However, it remains to be used reliably in these samples.

Single cell analysis has been revolutionized biomedicine research and provide further insight of the biology. ${ }^{11,12}$ Single cell transcriptome analysis, for example, has been well established to decipher the gene expression, as well as epigenetic regulation in games as well as preimplantation embryos in mice and human. ${ }^{11,13}$ Single cell proteomic, on the other hand, has been lag behand for the technical challenge. Nevertheless, the single cell analysis of immune cell and another cell have proceeded very rapidly. Our previous study has shown that single cell analysis has shown great potential in immune cell subtyping, and cancer cell heterogeneity analysis. ${ }^{3,14}$ However, until now, no study has been used in the embryo quality analysis.

In the current study, to further understand the relationship of embryo quality, implantation potential with the cytokines, we used the single cell proteomics, which was developed in our previous study. Our study has shown that the embryo culture medium showed different value of cytokines in embryo. Furthermore, we have shown the important difference of TNF-a between different quality of embryos. Using immunofluorescence stain, we have found differences of localization of TNF-a in these embryos. All the results proved further evidence that TNF-a expressed in embryo play critical roles in embryo invasion, and dysregulation. This factor has great impact for embryo implantation potential during IVF process.

\section{Materials And Methods}

\subsection{Patients}

Infertile couples undergoing IVF-ICSI treatment with fresh cleavage embryo transfer at our center from July 2018 were included in this study. The indications for ART were tubal factor infertility, anovulatory, endometriosis, male factor infertility, or unexplained. In cases of male subfertility, immunologic factors, or unexplained fertilization failure in a previous IVF cycle, we performed ICSI. No patient selection or exclusion criteria were used. Our study was approved by the ethics committee of the Reproduction \& women-children Hospital of Chengdu University of Traditional Chinese Medicine, and all patients had given informed consent. 


\subsection{Single cell proteomic cytokine measurement}

We collected culture medium on the day of transfer of each embryo and conduct measurement of cytokine levels. The detailed methods have been described in the previous study. ${ }^{3}$ The PDMS microchips with paralleled microchannels (100 $\mu \mathrm{m}$ width) was assembled with a poly-L-lysine coated glass slide. Different capture antibodies ( $1.5 \mu \mathrm{l}$ for each antibody) was injected into the individual microchannels for overnight coating to form antibody stripes for multiplexed protein detection. The microchannels were then washed with 1\% BSA 3 5 times and blocked with 3\% BSA for 10 min. After blocking, the PDMS microchip was removed, and the antibody-coated glass slide was washed with Dulbecco's phosphatebuffered saline (DPBS,), 50/50 DPBS/distilled (DI) water, and DI water sequentially before blowing dry with $\mathrm{N}_{2}$. Another PDMS microchip with paralleled microchannels (100 $\mu \mathrm{m}$ width) was treated with $\mathrm{O}_{2}$ plasma for 2 min to make it hydrophilic before aligned perpendicularly with antibody stripes on the glass slide. The microchannels were blocked with $3 \%$ BSA for $1 \mathrm{~h}$ to reduce nonspecific adsorption. Each sample $(1 \sim 2 \mu$ l) was injected into the individual microchannel to incubate overnight. After that, a mixture of detection antibodies and streptavidin-APC were added sequentially to react for 1 hour and 0.5 hour respectively to finish standard ELISA immunoassay procedures and obtain detectable sandwich complex. The fluorescence results were scanned and analyzed with GenePix 4300A scanner and its corresponding software (Molecular Devices, U.S.).

\subsection{Human ovarian stimulation protocols and oocyte retrieval}

Conventional ovarian stimulation for IVF-ICSI was performed. Patients were treated with standard gonadotrophin stimulation protocol using a short-acting gonadotropin-releasing hormone agonist ( $\mathrm{GnRH}$ a, Ferring, Switzerland), GnRH-a was administered at $0.1 \mathrm{mg} / \mathrm{d}$ from the middle luteal phase of the last menstrual cycle to desensitize the pituitary gland. With the long-acting gonadotropin-releasing hormone agonist (GnRH-a, Diphereline, IPSEN), people were administered with $3.75 \mathrm{mg} \mathrm{GnRH-a}$ at the menstrual phase. And for the antagonist protocol, patients were treated at $0.25 \mathrm{mg} / \mathrm{d}$ of gonadotropin-releasing hormone antagonist (GnRH-A, Cetrotide, Merck Serono). Ovarian stimulation was started with doses of follicle-stimulating hormone (recombinant FSH, Gonal-f; Merck Serono) or human menopausal gonadotropin (Livzon Pharmaceutical Group) varying from 150-300 IU/day according to the patient's age and ovarian reserve. The dose of recombinant FSH and human menopausal gonadotropin was adjusted and individualized for each patient based on the follicular growth. Ovarian stimulation was monitored by transvaginal ultrasound and measurement of estradiol plasma levels. An intramuscular injection of human chorionic gonadotropin (hCG, Livzon Group) at 5000-10,000 U was administered during the night when the follicles $\geq 18 \mathrm{~mm}$ in diameter accounted for more than half of those $\geq 14 \mathrm{~mm}$, and the average estradiol level of the follicles $\geq 14 \mathrm{~mm}$ in diameter was not less than $200 \mathrm{pg} / \mathrm{ml}$ for the $\mathrm{GnRH}$-a protocol. The same doses hCG and time was administered for GnRH-A protocol when the follicles $\geq 18 \mathrm{~mm}$ in diameter accounted for more than two or $\geq 17 \mathrm{~mm}$ in diameter accounted for more than three. 
Ultrasound-guided puncture was conducted for oocyte retrieval approximately 36 hour later. All oocyte retrieval and embryo transplantation surgeries were performed by the same experienced surgeon and embryos were scored as described by Gardner et al. ${ }^{15}$ The number of transplanted embryos was no more than two each time and was generally two. An intramuscular injection of progesterone $(80 \mathrm{mg} / \mathrm{d}$; Zhejiang Xiangju Pharmaceutical Co., Ltd) was given for luteal support.

\subsection{Embryo immunofluorescence stain methods}

The embryo was fixed with 4\% PFA for 15 min at room temperature and add $0.3 \%$ Triton X-100 in PBS for 20 min to change cell membrane permeability. Then the embryo was incubated with TNF-a primary antibody (1:100, Proteintech, Inc.) and CDX2 primary antibody (1:100, Abcam, Inc), at $4^{\circ} \mathrm{C}$ overnight. Subsequently, the embryo was incubated with alexa fluor 594-conjugated goat anti-mouse $\lg \mathrm{G}(\mathrm{H}+\mathrm{L})$, (1:1000, Invitrogen, Inc.) and 488-conjugated goat anti-rabbit IgG $(H+L),(1: 1000$, Invitrogen, Inc.), for 60 min at room temperature in dark place, followed by mounting with antifade mounting medium with DAPI (Beyotime, Inc., Shanghai, China). Then, analysis was conducted using a confocal laser scanning microscope (Olympus FV1000, Olympus Corporation) (Nikon N-STORM \& A1, Nikon Corporation). The six embryos used for staining came from the same volunteer.

\subsection{Ishikawa cell culture}

Human endometrial cancer cell line Ishikawa was purchased from the Biovector NTCC (Beijing, China). Cells were cultured in Eagle's minimum essential medium (EMEM; ATCC; Manassas, VA, USA) supplemented with 10\% (V/V) FBS (Gibco; Thermo Fisher Scientific, Inc.) and $100 \mathrm{nM}$ penicillin/streptomycin in a $5 \% \mathrm{CO}_{2}$ incubator at $37^{\circ} \mathrm{C}$.

\subsection{Cell counting kit-8 analysis}

In each well of a 96-well plate, $1 \times 10^{4}$ Ishikawa cells were cultured as attached monolayers overnight. Then different dosage of TNF-a (R\&D, Minneapolis, MN, USA) and ETA were used to treat the cell line. After 48 hours treatment, photos were taken using an inverted microscope (Olympus IX71, Olympus Corporation), and cells processed with the cell counting kit-8 from Dojindo (Shanghai, China). Quantitative analysis was investigated using the automatic microplate reader (varioskan flash, Fisher Scientific, Inc.). Each test had two replicates $(n=3)$.

\subsection{Apoptosis measurement with TUNEL staining}

Ishikawa cells were grown on $14 \mathrm{~mm}$ glass coverslips at a density of $2 \times 10^{5}$ cells per well of a 24-well plate. Then $10 \mathrm{ng} / \mathrm{ml} \mathrm{TNF-a}$ and $25 \mu \mathrm{g} / \mathrm{ml}$ ETA were used to treat the cell line. After 48 hours treatment, 
cells were analysis with Tunel BrightGreen Apoptosis Detection Kit (Vazyme, Inc., Nanjing, China), followed by mounting with antifade mounting medium with DAPI (Beyotime, Inc., Shanghai, China). Then, analysis was conducted using a confocal laser scanning microscope (Olympus FV1000, Olympus Corporation). The DNase $\otimes$ (Vazyme, Inc., Nanjing, China) treated control group cells served as positive control. Each test had two replicates $(n=3)$.

\subsection{Reverse transcription-quantitative poly-merase chain reaction (RT-qPCR)}

In each well of a 6-well plate, $2 \times 10^{5}$ Ishikawa cells were cultured as attached monolayers overnight. Then $10 \mathrm{ng} / \mathrm{ml} \mathrm{TNF-a} \mathrm{and} 25 \mu \mathrm{g} / \mathrm{ml}$ ETA were used to treat the cell line. After 6hour, 24 hour or 48 hour treatment, cells were collected to analysis with RT-qPCR. Each test had two replicates $(n=3)$. Cell total RNA isolation kit (Foregene, Inc., Chengdu, China) was used to isolate total RNA from Ishikawa cells, according to the manufacturer's protocol. Furthermore, a total of $2 \mu \mathrm{g}$ RNA sample was reverse-transcribed into $\mathrm{CDNA}$ at $42^{\circ} \mathrm{C}$ for 30 min using the all-in-one $\mathrm{CDNA}$ synthesis supermix (Bimake, Inc., Houston, TX, USA), and the qPCR assay was performed with $0.25 \mu \mathrm{l}$ CDNA using $2 \mathrm{X}$ Supermix (Bimake, Inc., Houston, TX, USA) using the CFX96 Touch System (Bio-Rad Laboratories, Inc.). The data was analyzed using CFX Manager software (version 3.0; Bio-Rad Laboratories, Inc.) and $\beta$-actin gene expression was used as an internal reference. Data was analyzed using the $2-\Delta \Delta \mathrm{Cq}$ method. The following primer sequences were used for the PCR: Caspase8, forward, 5'- GTT GTG TGG GGT AAT GAC AAT CT -3' and reverse, 5'- TCA AAG GTC GTG GTC AAA GCC -3'; TNFR1, forward, 5'- TCA CCG CTT CAG AAA ACC ACC -3' and reverse, 5'- GGT CCA CTG TGC AAG AAG AGA -3'; CRISP2, forward, 5'- AGG ATC CCG CTT TTA CTG CT -3' and reverse, 5'- TGT TAC TGG CAG GTG GAG AG -3'; AQP3, forward, 5'- GGG GAG ATG CTC CAC ATC C -3' and reverse, 5'- AAA GGC CAG GTT GAT GGT GAG -3'; ENaCA, forward, 5'- TCT GCA CCT TTG GCA TGA TGT -3' and reverse, 5'- GAA GAC GAG CTT GTC CGA GT -3'; CFTR, forward, 5'ATG GGA GAA CTG GAG CCT TC -3' and reverse, 5'- CTC GTT GAC CTC CAC TCA GT -3'; LIF, forward, 5'CCA ACG TGA CGG ACT TCC C -3' and reverse, 5'- TAC ACG ACT ATG CGG TAC AGC -3'; CRYBB2, forward, 5'- CAA CTT CAC CGG GAA GAA GAT G -3' and reverse, 5'- CGG GGT ACT GGT AGC CAA C -3', and $\beta$-actin, forward, 5'- CAT GTA CGT TGC TAT CCA GGC -3' and reverse, 5'- CTC CTT AAT GTC ACG CAC GAT -3'. The following PCR conditions were used for PCR: Initial denaturation for 1 min at $95^{\circ} \mathrm{C} ; 40$ cycles of $95^{\circ} \mathrm{C}$ for $10 \mathrm{sec}$ and $60^{\circ} \mathrm{C}$ for $20 \mathrm{sec}$.

\subsection{Statistical analysis}

All results are presented as the mean \pm standard error of the mean. Statistical analysis was performed using GraphPad Prism 7 Project software (GraphPad Software Inc., San Diego, CA, USA). Quantitative image analysis was made using Image-Pro Plus 6.0 software (Media Cybernetics, Rockville, MD). Differences were analyzed using one-way analysis of variance followed by the Newman-Keuls post hoc test. $P<0.05$ was considered to indicate a statistically significant difference. 


\section{Results}

\subsection{TNF- $a$ is significantly increased in the non-pregnancy group}

In the current study, we have recruited the two groups from IVF center and the clinic characteristics were shown in the Table 1. As shown in the result, the median age of the nonpregnancy is older compared with the live birth group $(P<0.05)$, and the duration of infertility is higher in nonpregnancy group. The other parameters show no significant difference between the two groups. In the current result, we investigated the major Th1/Th2 related cytokines from the spent medium of IVF. And our result showed that of the 10 cytokines we analyzed, which were IL-1 $\beta$, IL-6, IL-8, IL-10, MCP1, MIP-1a, MIP-1 $\beta$, RANTES, GMCSF and TNF-a, in group of implantation failure, only TNF-a levels were significantly increased, compared with the group of control group (Figure 1A-J). Other cytokines show no significant difference between implantation group and group of implantation failure, indicating that TNF-a is the most significantly changed cytokines relevant to the implantation potential. The TNF-a ROC curve showed the area under the curve equals 0.8095 (Figure 1K). Interestingly, the association analysis indicated that the level of TNFa is significantly associated with age (Figure 1L), Therefore, our result indicates that the advanced age could contribute to higher TNF-a concentration in embryo culture medium, and higher TNF-a maybe highly relevant to the implantation failure of the embryo.

Table 1

Characteristics of nonpregnancy and pregnancy from IVF center.

\begin{tabular}{|lll|}
\hline CHARACTERRISDIC & OUTCOME & \\
\hline & NONPREGNANCY & PREGNANCY \\
\hline $\mathrm{N}(\%)$ & 12 & 9 \\
\hline Age (year), median & 36.5 & 28 \\
\hline Duration of infertility, median (IQR) & $7.0(4-11.25)$ & $4.0(2.75-4.5)$ \\
\hline Primary infertility & $6(50)$ & $6(66.67)$ \\
\hline Cause of infertility & & \\
\hline$>1$ cause & $6(50)$ & $1(0.11)$ \\
\hline Tubal & $4(33.33)$ & $6(66.67)$ \\
\hline Anovulatory & 0 & 0 \\
\hline Male factor & $2(0.17)$ & $2(22.22)$ \\
\hline Endometriosis & 0 & 0 \\
\hline Unexplained & 0 & 0 \\
\hline
\end{tabular}




\subsection{The immunofluorescence stain of TNF-a indicated polarized localization in early human embryo}

We found that TNF-a is the most significantly up-regulated cytokine in the spent culture medium coincide with previous researches imply TNF-a stay a vital position in implantation, consider embryo attached endometrium with a specific pattern, we then used immunofluorescence to detect whether TNF-a expression has unique characteristic.

Our result showed that the TNF-a is indeed expressed in both the ICM and the trophectoderm (TM) of the embryo. Interestingly, the TNF-a polarized expression pattern was found (Figure 2A). Advanced, we found low quality embryo has a typically dispersed distribution (Supplementary figure 1). On the other hand, clinical symptom showed that poor quality embryo is one of the major reasons leading to implantation failure. According to our result, we found the fluorescent intensity of TNF- $a$ is negative correlated to embryo quality, especially ICM score (Figure 2B, C). It indicated TNF-a expression is a monitor of embryo quality and probably interferes in implantation process.

\subsection{TNF-a treatment lead to inhibited proliferation, increased apoptosis in Ishikawa cell line}

To further delineate whether increased TNF-a is detrimental for the embryo implantation, we examine whether adding TNF-a could affect the endometrium receptivity. Increased apoptosis and decreased proliferation are the two significant features related to endometrium receptivity. We check whether TNF-a affects apoptosis of the endometrium cell. Then Ishikawa cell were treated with $0 \mathrm{ng} / \mathrm{ml}, 1 \mathrm{ng} / \mathrm{ml}, 2$ $\mathrm{ng} / \mathrm{ml}, 5 \mathrm{ng} / \mathrm{ml}, 10 \mathrm{ng} / \mathrm{ml}$ and $20 \mathrm{ng} / \mathrm{ml}$ of TNF-a independently for 48 hours (Figure 3C). We then chose $10 \mathrm{ng} / \mathrm{ml}$ TNF-a for subsequent experiments, which could cause cell damage significantly. And our result showed that adding $10 \mathrm{ng} / \mathrm{ml}$ of TNF-a lead to increase apoptosis in Ishikawa cell line, which could be inhibited by ETA, a well-known TNF-a inhibitor (Figure 3A, B). After treatment of TNF-a inhibitor, ETA, the proliferation cell number is also significant rescued compared with the TNF-a treatment alone (Figure 3C, D).

\subsection{TNF-a treatment could activate TNF- $a$ receptor and apoptosis related genes in Ishikawa cell line}

If excessive TNF-a is detrimental for endometrium receptivity through regulation of apoptosis and proliferation, it may affect these changes through mediating of TNF-a receptor mediated apoptosis in endometrium cell. We then checked the expression of TNF-a receptor as well as apoptosis related genes. 
Our result showed that TNFR1 and Caspase8 expression is significantly increased. Ion channel such as $\mathrm{ENaCA}$ and CFTR are ion channels with important function in regulating endometrium, which has been shown to critical for endometrium receptivity (Figure 4). Our experiment showed that adding TNF-a lead to up-regulation of ion channel gene expression. We also check the other receptivity marker, such as LIF, and our result showed that add TNF-a do increase the expression of LIF (Figure 4).

If the TNF-a could secrete from the embryo, then whether blocking the TNF-a mediated with ETA, a proven TNF-a antagonist, and has been shown to block TNF- $a$ activation, can rescue the phenotype caused by TNF-a secreted from the embryo? Our results do show that add ETA could significantly inhibit the gene expression, apoptosis cell numbers compared with TNF-a group, indicating blocking the TNFR $1 / 2$ can significantly inhibit the apoptosis caused by the TNF-a (Figure 3A, B). Together, the result showed that excessive TNF- $a$ is detrimental for the endometrium receptivity through activation of TNF-a receptor and down-stream pathway.

\section{Discussion}

In our study, we have shown that TNF-a is a critical factor secreted by the embryo and the secretion is significantly increased in the embryo culture medium in implantation failure patients compared with control. The higher level of spent medium from the embryo of implantation failure shown that TNF-a levels as important determinants of implantation potential.

The source of TNF-a in embryo is an interesting question. The TNF-a is either secreted from the embryo, or it can be expressed from the sperm or oocyte. ${ }^{16}$ The single cell sequencing project has rapidly accelerated the detection. ${ }^{13,17}$ In our database, we have found one subgroup showed expression of TNFa. However, there is no expression in the oocyte database. Therefore, the exact source of TNF-a remains further investigation. Furthermore, we do not find the expression in the embryo database. On the other hand, the expression of embryo induced genes, including TNFR1 expression is increased. All this result indicated that TNF-a expression in normal embryo is quite low, if not stimulated in the embryo. One interesting observation is that the association study showed the significant association of age and the value of TNF-a, indicating that understanding the impact of older age to embryo quality could helpful to further investigate the source of higher secretion in embryos of implantation failure.

In the previous study, some researchers have found that some cytokines, such as II-8 has shown differential expression between implanted and non-implanted embryos. However, the method used and the detection thread is low in this study. Although our method is semi-quantitative, and cannot have absolute quantification with the current method, our method can be applied to absolute quantification with a caliber with revision.

One interesting observation is that, in our study, some cytokines, such as GMCSF, IL-1 level also showed increased expression in non-implanted embryos. GMCSF is also important for embryo implantation, and recent observation showed that uterine infusion of cytokine, such as CSF showed successful outcome to 
treat of repeated miscarriage patients. ${ }^{18}$ Another potential implication of the current study is that recent study has showed the cytokines are beneficial for the growth of poor-quality embryo. All these studies indicated that the exact mechanism of how cytokines regulate embryo implantation need further investigation.

The non-invasive measurement of biomarker is currently under intensive studied, and so far, some groups have showed great progress. Except for the protein marker, other nucleic acid, and other markers have been studied. ${ }^{19,20}$ Other methods could also show valuable use in predicting the potential of the embryo. ${ }^{6,21}$ It is hopeful that together with biomarker, the future study would provide more reliable biomarker during IVF process.

How the increased TNF-a contribute to the implantation failure. Several points need to clarify the underlying mechanism. First, the increased TNF-a could lead to the apoptosis through the binding to the receptor. TNF-a has two receptors, and it has been shown that it can bind to TNF-a receptor to activate the down-stream process. ${ }^{20}$ Second, the increased secretion would harmful to the endometrium. Other potential mechanism also contributed to the secretion of TNF-a. For example, recent study has shown that ATP is involved in the secretion of TNF-a from membrane. ${ }^{8,22}$ Thirdly, the down-stream target of TNFa warrant further clarification. Secretion of excessive TNF-a increased the expression of pyroptosis marker, Caspase-8, which may indicate the possibility that excessive TNF-a leads to the pyroptosis of the embryo and endometrium, leading to the demise of the embryo during implantation window. ${ }^{23}$ Interestingly, our study has showed that TNF-a could also increase the expression of implantation marker, such as LIF, and other markers. It is believed that the in vivo study would clarify the exact mechanism. TNF-a blocker such as, ETA have been widely used for the prevention of miscarriage. ETA is a fusion protein of the TNF receptor and IgG1 Fc, and has been widely used as clinical TNF-a inhibitor in autoimmune diseases such as rheumatoid arthritis, et.al. ETA showed excellent ability to scavenge TNF-a molecules in vitro as well as in vivo, and our previous study has shown that it could activate NF-KB signaling pathway in ovary during PCOS pathogenesis. ${ }^{24}$ The underlying mechanism of how ETA could promote embryo implantation need further investigation. It is believed that the current study would be helpful to the future interventional study for cytokines in IVF treatment. It also sheds light on the underlying mechanism for the potential role of TNF- $a$ in regulating endometrium receptivity to promote the proper implantation of the embryo.

\section{Conclusions}

In conclusion, in the current study, we have found that increased secretion of TNF- $a$ is related to implantation failure and our study showed that blocking of TNF-a pathway could be a potential effective prophylactic treatment for this condition.

\section{Abbreviations}

TNF-a: Tumor necrosis factor alpha 
ICM: Blastocysts inner cell mass

TM: Trophectoderm of the embryo

ETA: Entanecept

TNFR1: Tumor necrosis factor receptor 1

TNFR2: Tumor necrosis factor receptor 2

CFTR: Cystic fibrosis transmembrane conductance regulator

ENaCA: Epithelial $\mathrm{Na}(+)$ channel subunit alpha

AQP3: Aquaporin-3

CRISP2: Cysteine-rich secretory protein 2

LIF: Leukemia inhibitory factor

CRYBB2: Beta-crystallin B2

Th1/2: Helper T cells 1/2

IL-1ß: Interleukin-1 beta

IL-6: Interleukin-6

IL-8: Interleukin-8

IL-10: Interleukin-10

MCP1: Monocyte chemotactic protein 1

MIP-1a: Macrophage inflammatory protein 1-alpha

MIP-1 $\beta$ : Macrophage inflammatory protein 1-beta

RANTES: C-C motif chemokine 5, T-cell-specific protein

GMCSF: Granulocyte-macrophage colony-stimulating factor

NF-kB: Nuclear factor of kappa light polypeptide gene enhancer in B-cells 3

IVF: In vitro fertilization

ICSI: Intracytoplasmic sperm injection 
ART: Assisted Reproductive Technology

ROC: Receiver operating characteristic curve

PCOS: Polycystic ovary syndrome

\section{Declarations}

\section{Ethics approval and consent to participate}

The Second Affiliated Hospital of Chengdu University of Traditional Chinese Medicine Ethics Committee; approval number: 2017-01. Participants provided consent to participate in this research.

\section{Consent for publication}

We promise to publish this article in Reproductive Biology and Endocrinology with exclusive quthorization.

\section{Availability of supporting data}

The dataset used and/or analyzed during the current study available from the corresponding author on reasonable request. Registry data are available publicly, see references.

\section{Competing interests}

None of the authors report any potential conflicts.

\section{Funding}

This work was supported by the National Key Research and Development Program of China (2018YFC1002804); Scientific and Translational Research Fund of Sichuan Provincial Department of Science and Technology (2017YSZH0032); The Key Research and Development Support Program of Chengdu (2019-YF05-00017-SN).

\section{Authors' contributions}

Wenming Xu and Minghui Zhu developed the concept of the study; all authors contributed to data accumulation; Jiao Lv and Xudong Shan contributed to data analysis; all authors contributed to data interpretation. Jiao Lv and Wenming Xu wrote the manuscript. All authors contributed to revisions of the 
manuscript, and approved of the final submission. Jiao Lv had full access to all the data in the study and takes responsibility for the integrity of the data and the accuracy of the data analysis.

\section{Acknowledgements}

We thank AIVFO (Inc., Chengdu, China) to provide technical support.

\section{References}

1. Szekeres-Bartho, J., S. Sucurovic, and B. Mulac-Jericevic. The Role of Extracellular Vesicles and PIBF in Embryo-Maternal Immune-Interactions. Front Immunol. 2018;9:2890.

2. Yang, M., et al. miR-15b-AGO2 play a critical role in HTR8/SVneo invasion and in a model of angiogenesis defects related to inflammation. Placenta. 2016;41:62-73.

3. Xue, Q., et al. Analysis of single-cell cytokine secretion reveals a role for paracrine signaling in coordinating macrophage responses to TLR4 stimulation. Sci Signal. 2015;8:59.

4. He, B., et al. Blastocyst activation engenders transcriptome reprogram affecting X-chromosome reactivation and inflammatory trigger of implantation. Proc. Natl. Acad. Sci. U. S. A. 2019;116:1662116630.

5. Coughlan, C., et al. Recurrent implantation failure: definition and management. Reproductive BioMedicine Online. 2014;28:14-38.

6. Jin, X.Y., et al. Pinopode score around the time of implantation is predictive of successful implantation following frozen embryo transfer in hormone replacement cycles. Hum. Reprod. 2017;32:2394-2403.

7. Horton, J., et al. Reproductive, obstetric, and perinatal outcomes of women with adenomyosis and endometriosis: a systematic review and meta-analysis. Hum. Reprod. Update. 2019;25:592-632.

8. Gu, X.W., et al. ATP mediates the interaction between human blastocyst and endometrium. Cell Prolif. 2019;e12737.

9. $\mathrm{Li}, \mathrm{X}$., et al. Ten-eleven translocation 2 demethylates the MMP9 promoter, and its down-regulation in preeclampsia impairs trophoblast migration and invasion. J. Biol. Chem. 2018;293:10059-10070.

10. Lee, M.S., et al. Simultaneous detection of two growth factors from human single-embryo culture medium by a bead-based digital microfluidic chip. Biosens Bioelectron. 2020;150:111851.

11. Li, L., et al. Single-Cell RNA-Seq Analysis Maps Development of Human Germline Cells and Gonadal Niche Interactions. Cell Stem Cell. 2017;20:858-873.

12. Li, L., et al. Single-cell multi-omics sequencing of human early embryos. Nat Cell Biol. 2018;20:847858.

13. Leng, L., et al. Single-Cell Transcriptome Analysis of Uniparental Embryos Reveals Parent-of-Origin Effects on Human Preimplantation Development. Cell Stem Cell. 2019;25:697-712. 
14. Ji, Y., et al. Multiplexed profiling of single-cell extracellular vesicles secretion. Proc. Natl. Acad. Sci. U. S. A. 2019;116:5979-5984.

15. Gardner, D.K., W.B.J.C.O.i.O. Schoolcraft, and Gynecology. Culture and transfer of human blastocysts. Current opinion in obstetrics \& gynecology. 1999;11:307-311.

16. Liu, B., et al. Telomere shortening by transgenerational transmission of TNF-a-induced TERRA via ATF7. Nucleic Acids Res. 2018;47:283-298.

17. Groff, A.F., et al. RNA-seq as a tool for evaluating human embryo competence. Genome Res. 2019;29:1705-1718.

18. Robertson, S.A., et al. Embryotoxic cytokines-Potential roles in embryo loss and fetal programming. $J$ Reprod Immunol. 2018;125:80-88.

19. Chegini, N., Q. Dou, and R.S. Williams. An inverse relation between the expression of tumor necrosis factor alpha (TNF-alpha) and TNF-alpha receptor in human endometrium. Am J Reprod Immunol. 1999;42:297-302.

20. Leaver, M. and D. Wells. Non-invasive preimplantation genetic testing (niPGT): the next revolution in reproductive genetics? Hum Reprod Update. 2020;26:16-42.

21. Law, T.S.M., et al. Endometrium imaging using real-time rotational optical coherence tomography imaging system: A pilot, prospective and ex-vivo study. Medicine (Baltimore). 2019;98:7738.

22. Soni, S., et al. ATP redirects cytokine trafficking and promotes novel membrane TNF signaling via microvesicles. Faseb j. 2019;33:6442-6455.

23. Günther, C., et al. Caspase-8 regulates TNF-a-induced epithelial necroptosis and terminal ileitis. Nature. 2011;477:335-339.

24. Lang, Q., et al. ETA-mediated anti-TNF-alpha therapy ameliorates the phenotype of PCOS model induced by letrozole. PLoS One. 2019;14:e0217495.

\section{Figures}


A

$\mathrm{IL}-1 \beta$
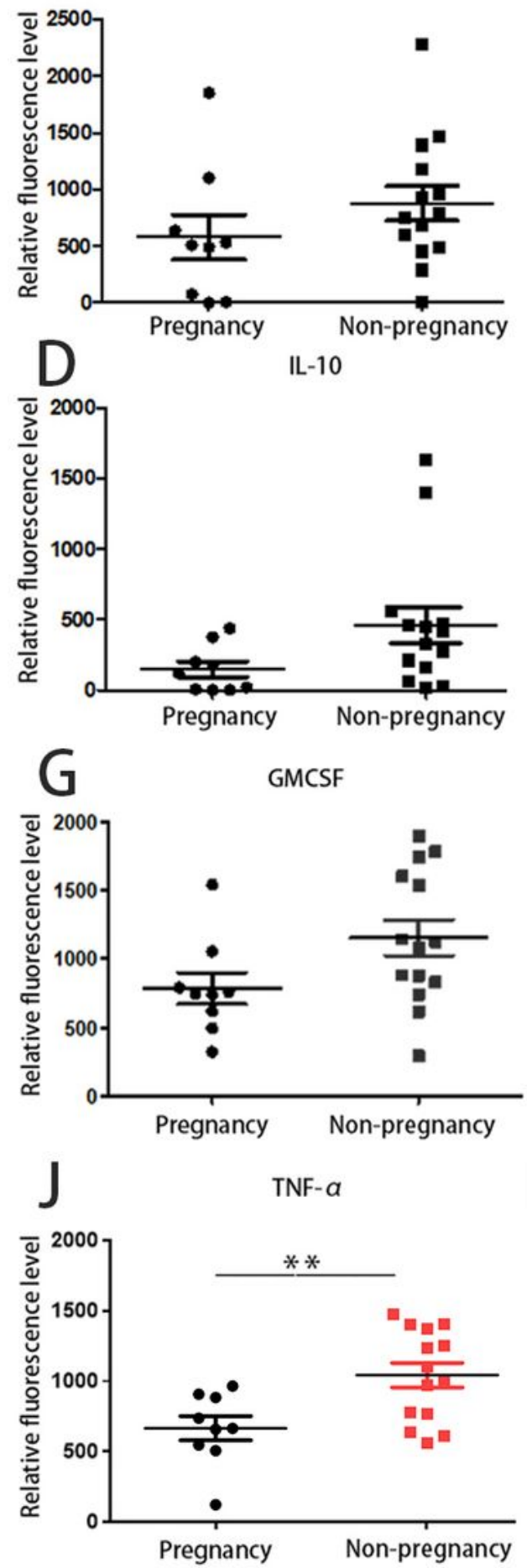

B
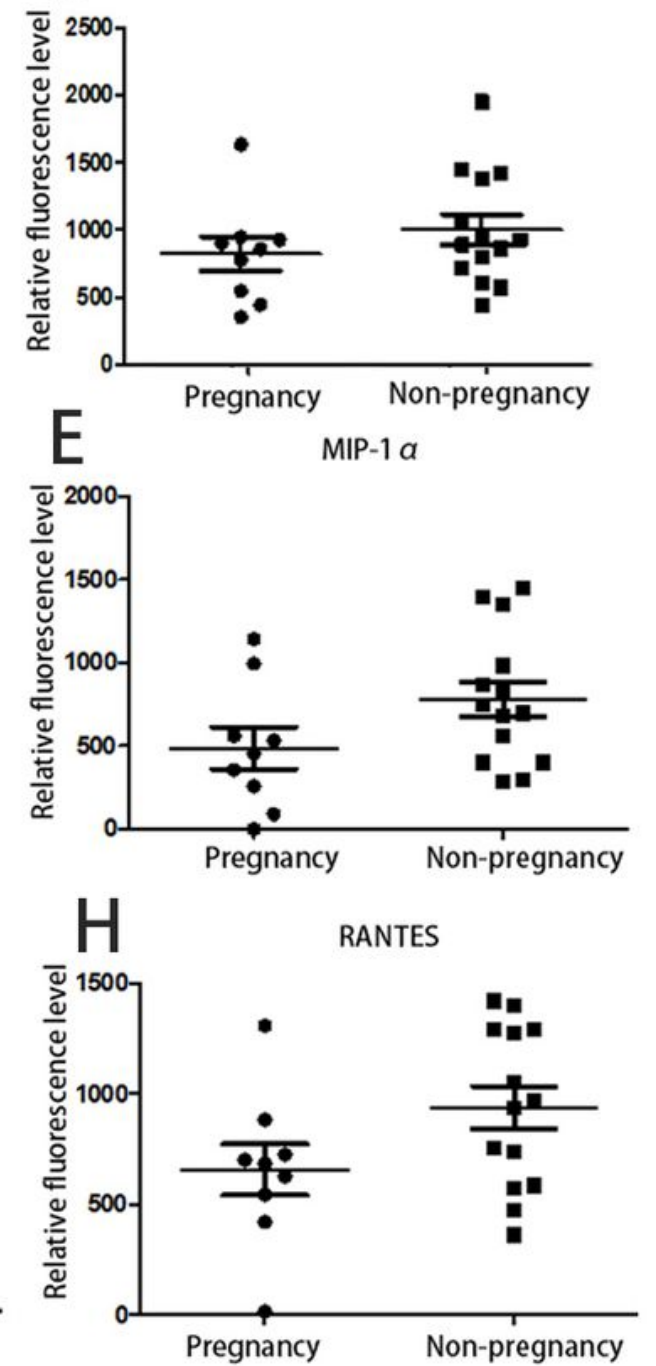

C

IL-8

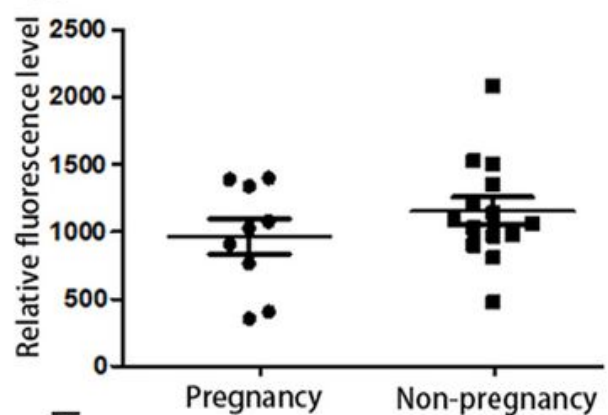

F MIP-1 $\beta$
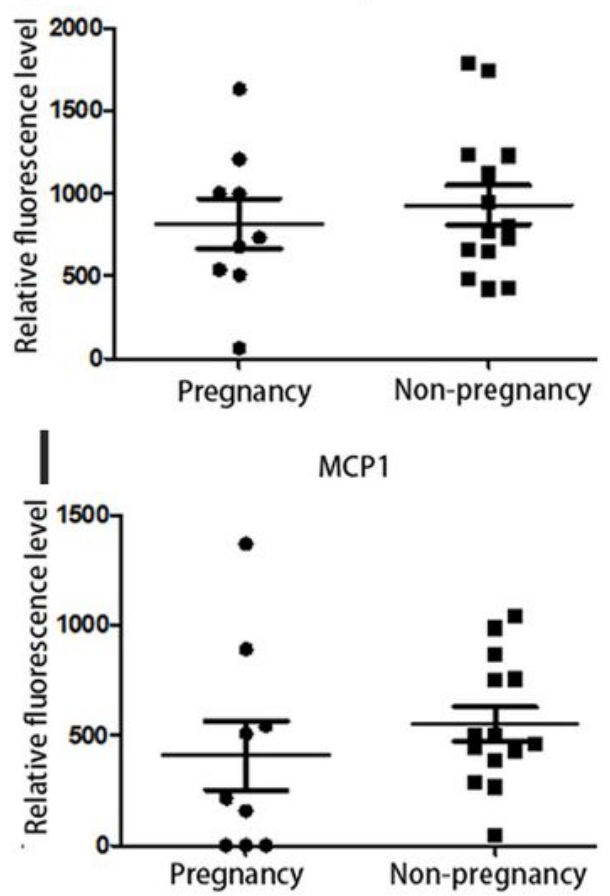

$\mathrm{K}_{100}$

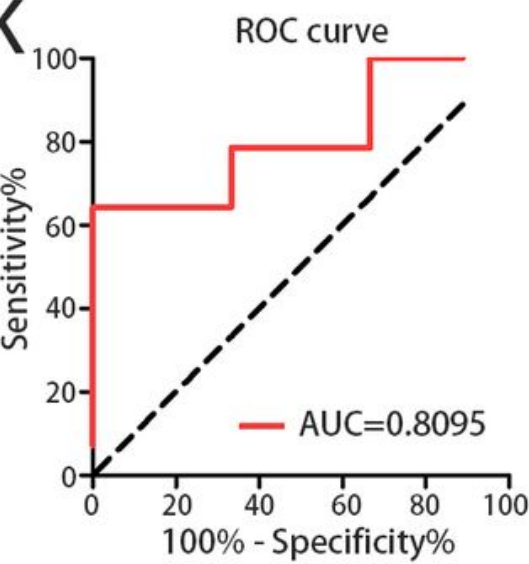

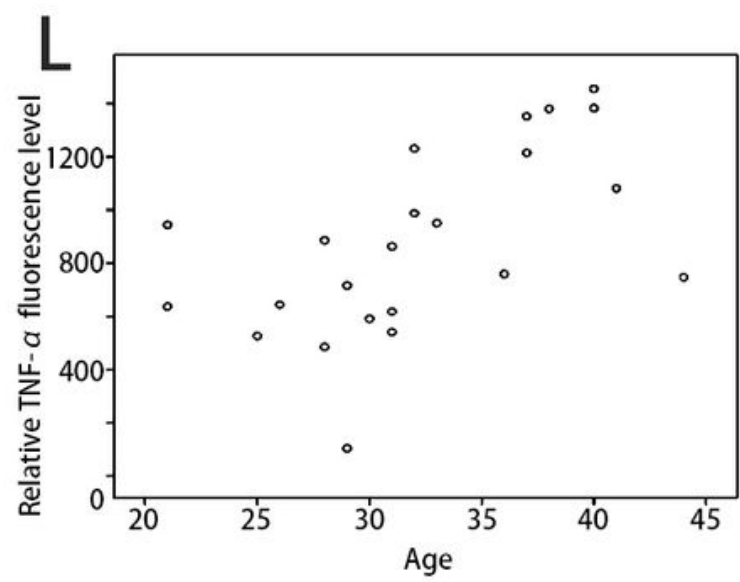

Figure 1

(A-J)10 cytokines in human embryo culture medium from IVF center were tested by the single cell cytokine array. ${ }^{*} \mathrm{P}<0.01$. Not all samples had signals at the time of testing. (K) The ROC curve of TNF-a. (L) Correlation analysis of age and TNF-a expression in culture medium. 
A

$\mathrm{IL}-1 \beta$
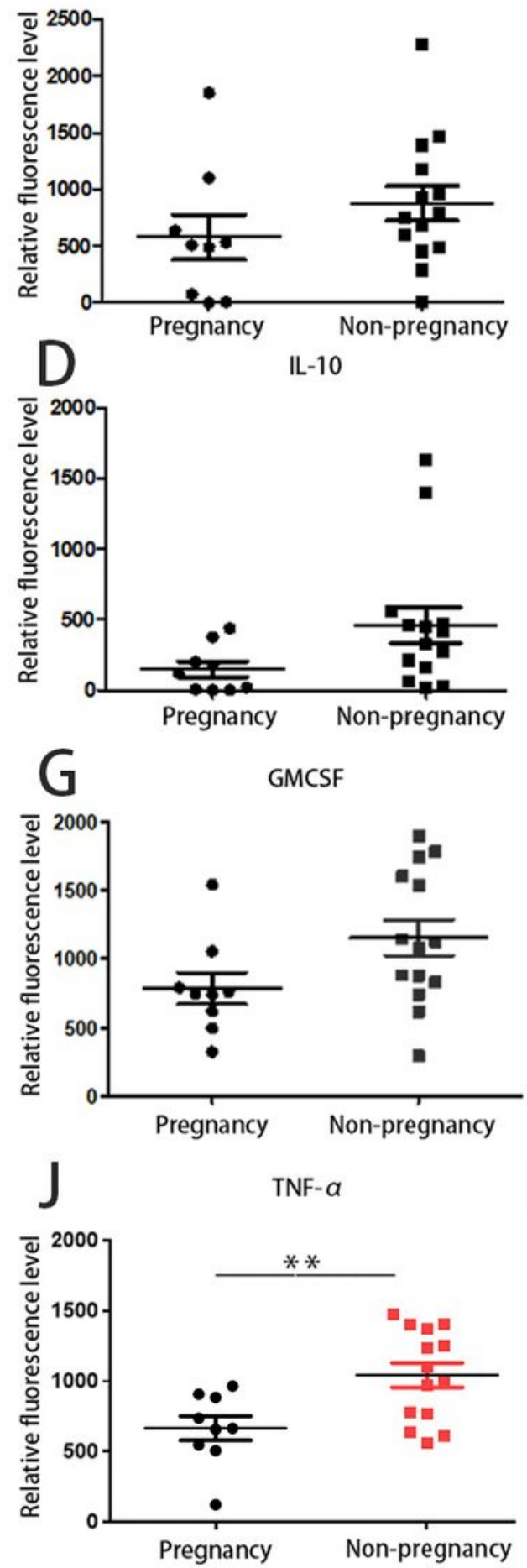

B
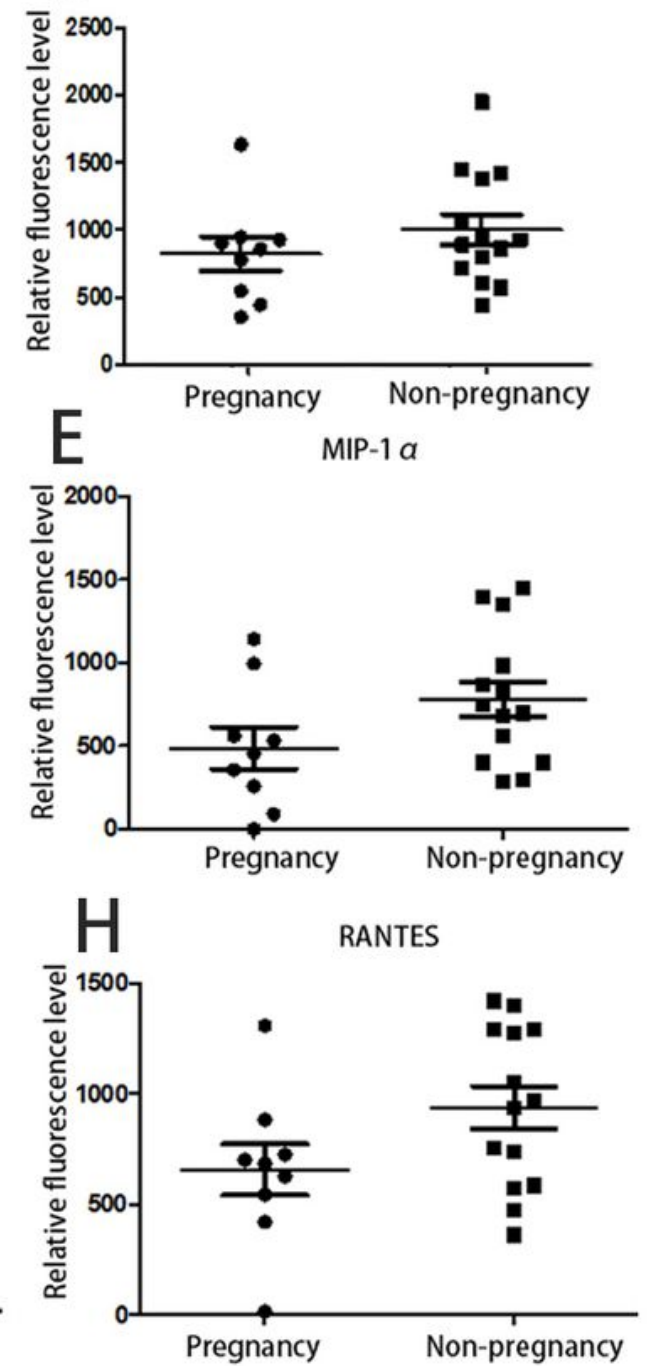

C

IL-8

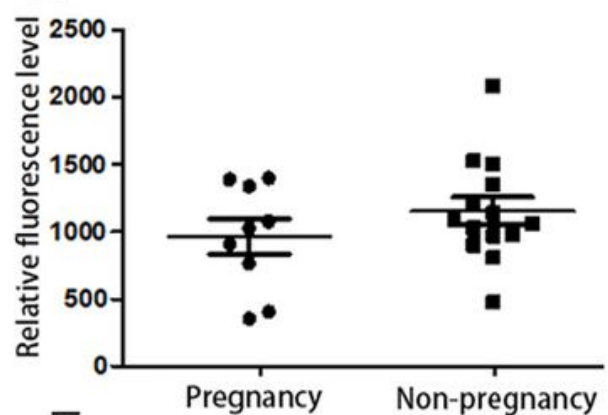

F MIP-1 $\beta$
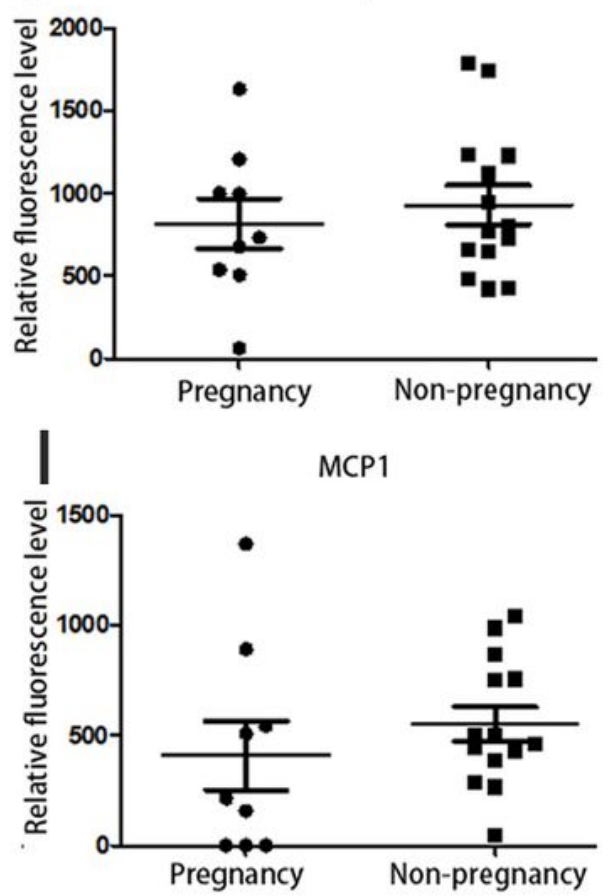

$\mathrm{K}_{100}$

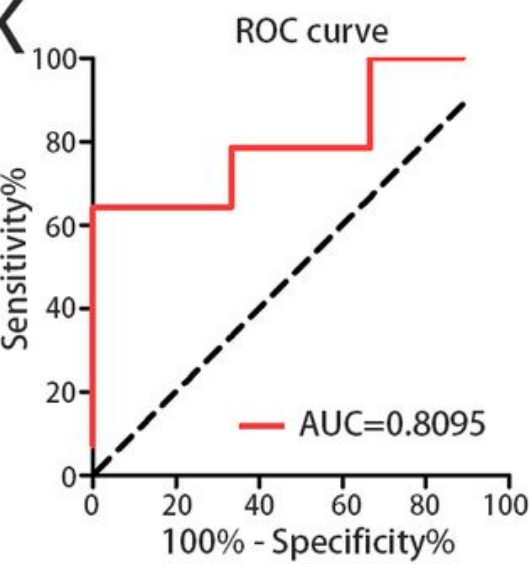

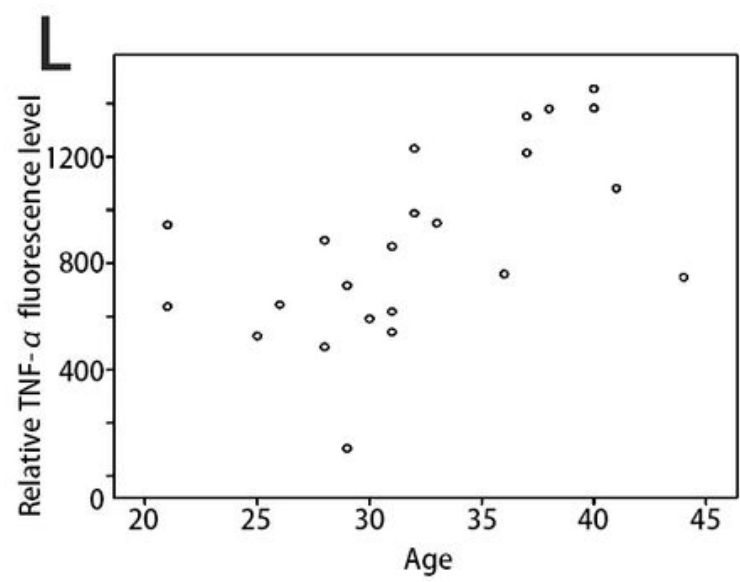

Figure 1

(A-J)10 cytokines in human embryo culture medium from IVF center were tested by the single cell cytokine array. ${ }^{*} \mathrm{P}<0.01$. Not all samples had signals at the time of testing. (K) The ROC curve of TNF-a. (L) Correlation analysis of age and TNF-a expression in culture medium. 
A

$\mathrm{IL}-1 \beta$
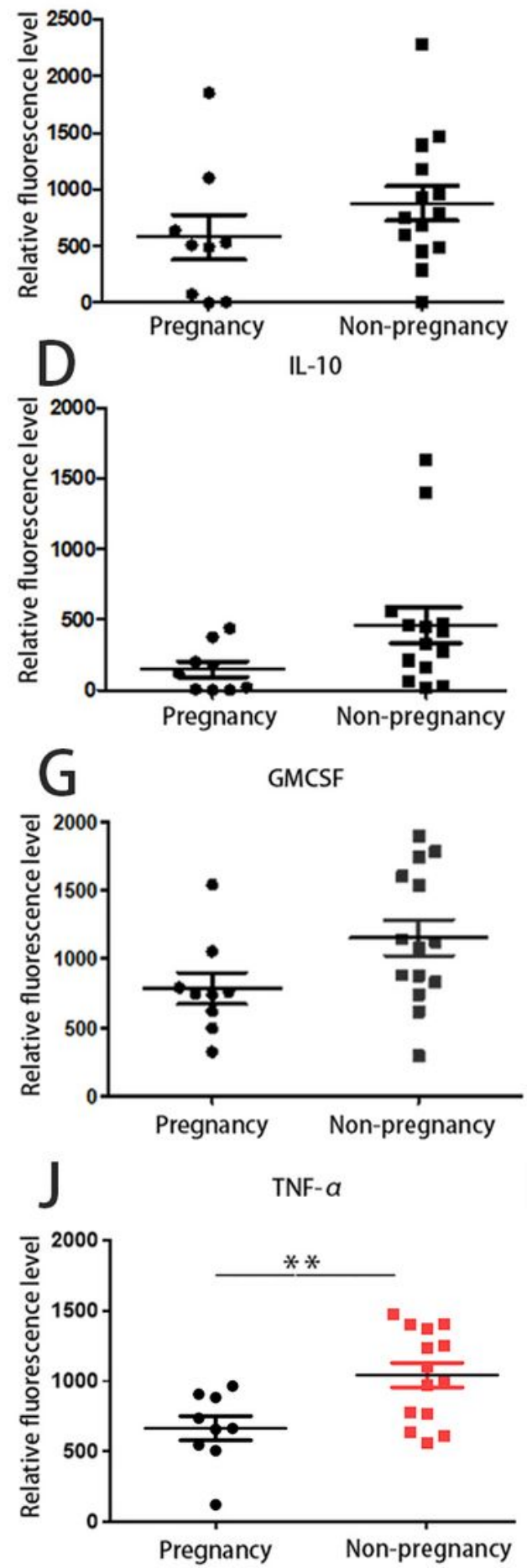

B
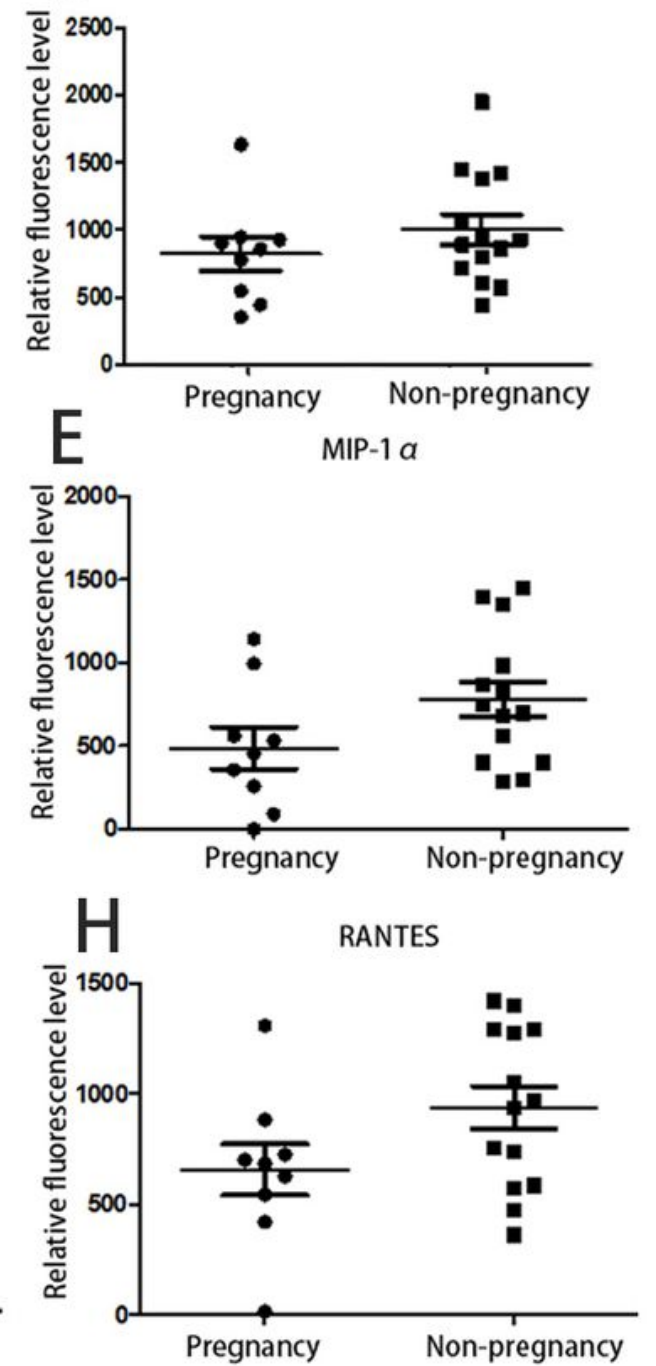

C

IL-8

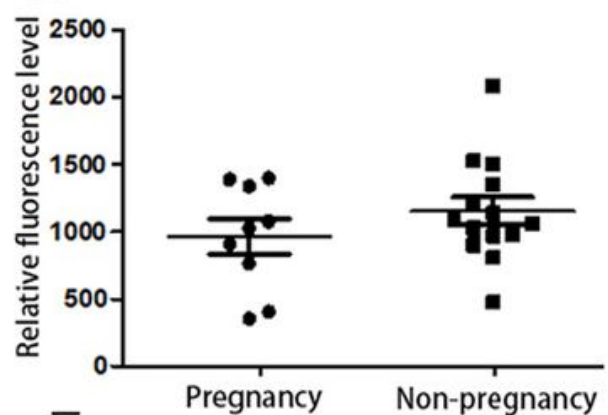

F MIP-1 $\beta$
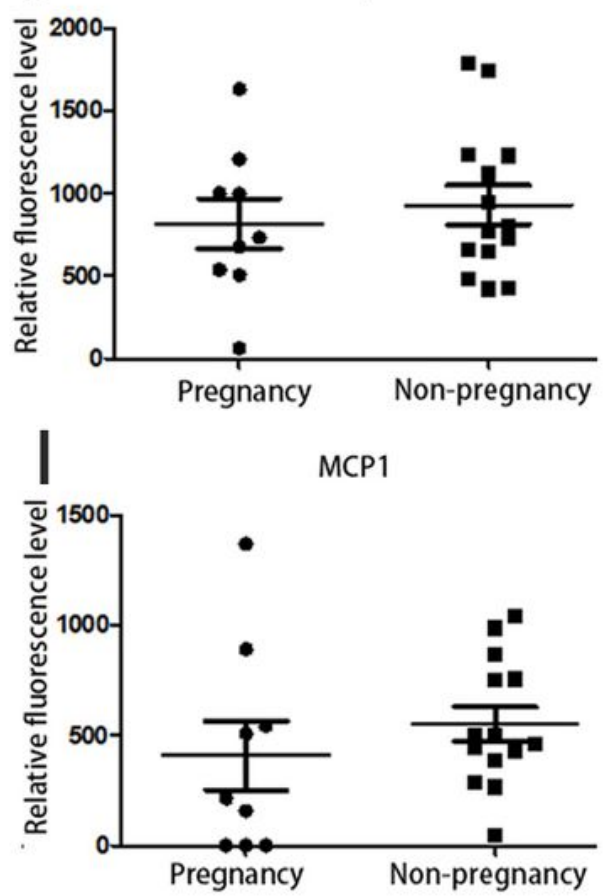

$\mathrm{K}_{100}$

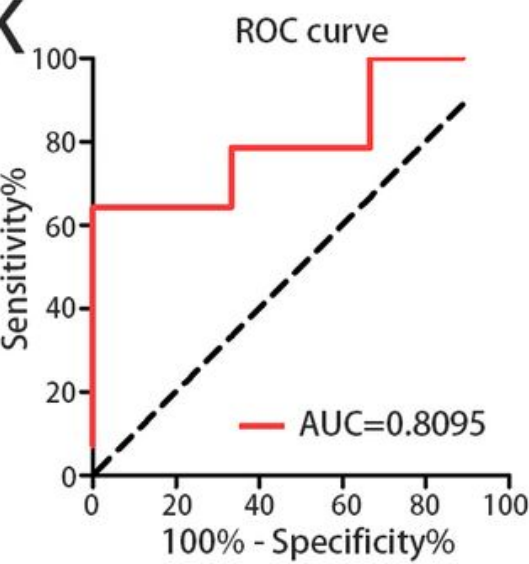

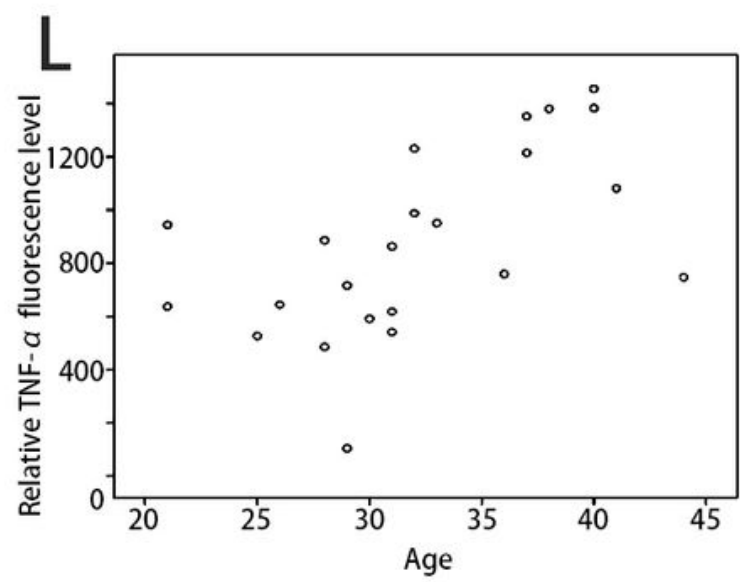

Figure 1

(A-J)10 cytokines in human embryo culture medium from IVF center were tested by the single cell cytokine array. ${ }^{*} \mathrm{P}<0.01$. Not all samples had signals at the time of testing. (K) The ROC curve of TNF-a. (L) Correlation analysis of age and TNF-a expression in culture medium. 
A

IL-1 $\beta$
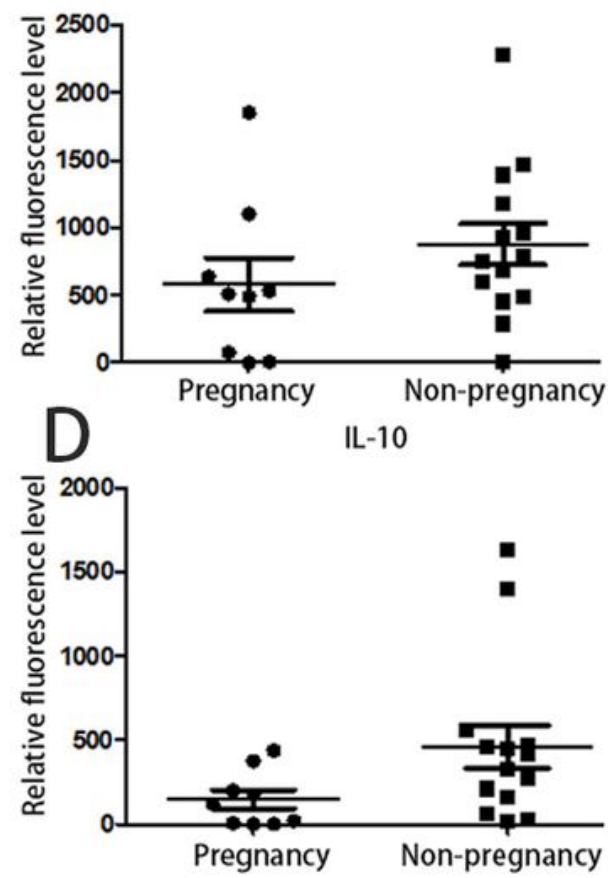

$G$
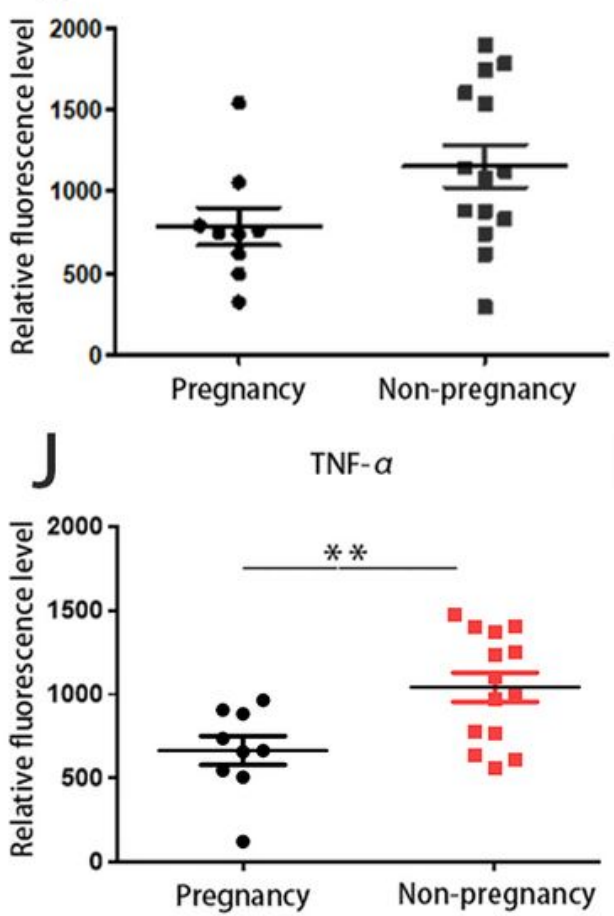

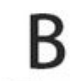

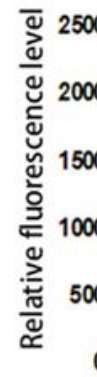

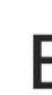

E
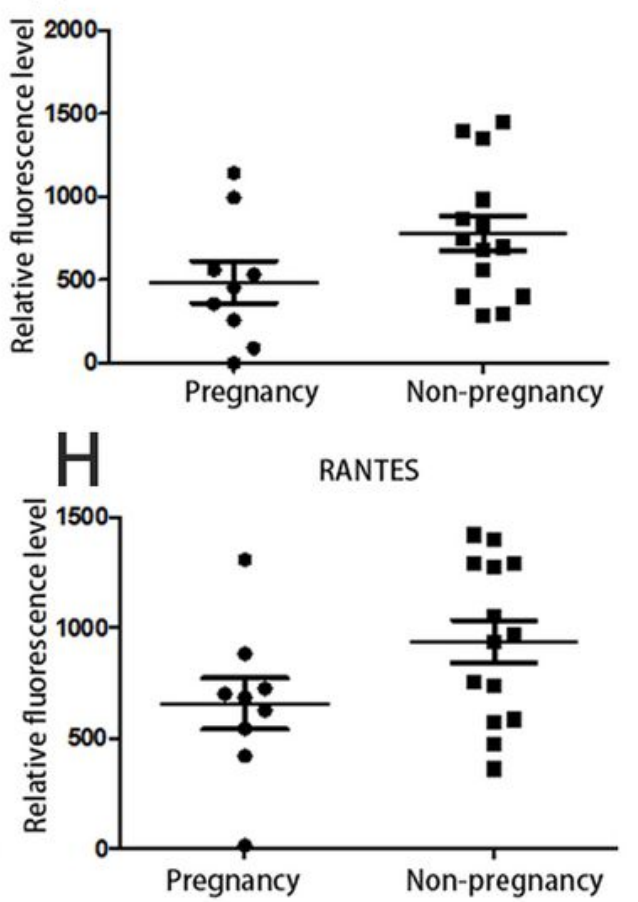

C

IL-8
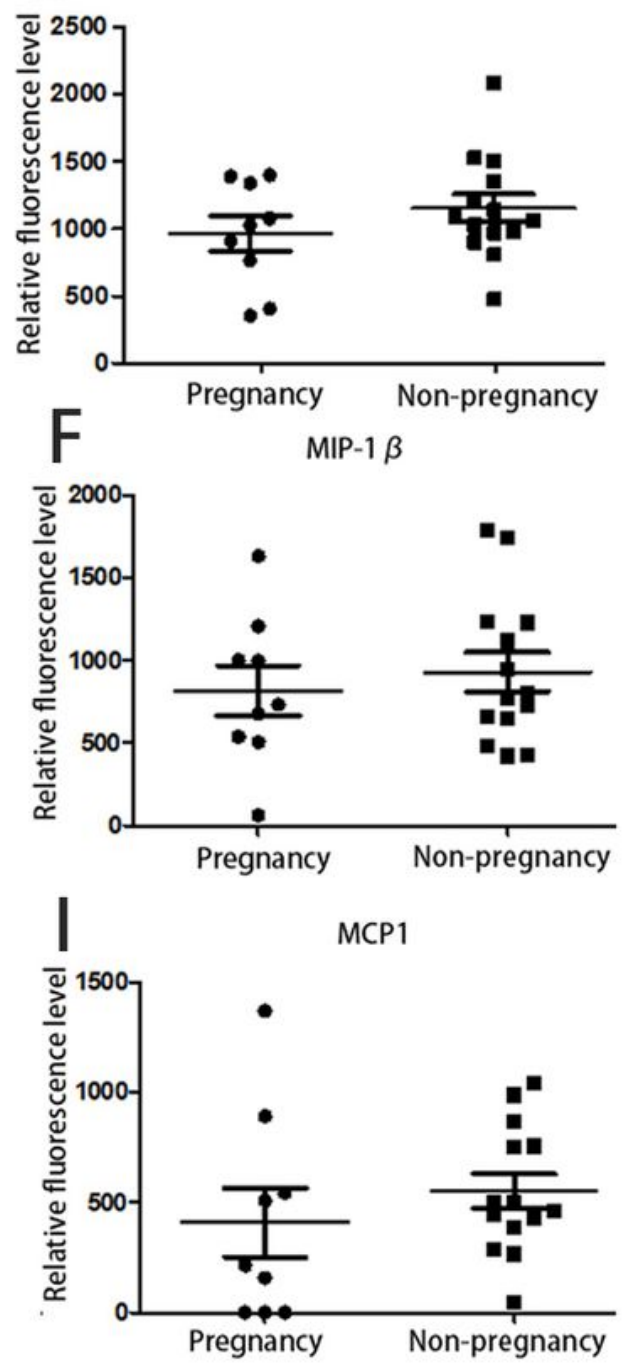

$\mathrm{K}_{100}$

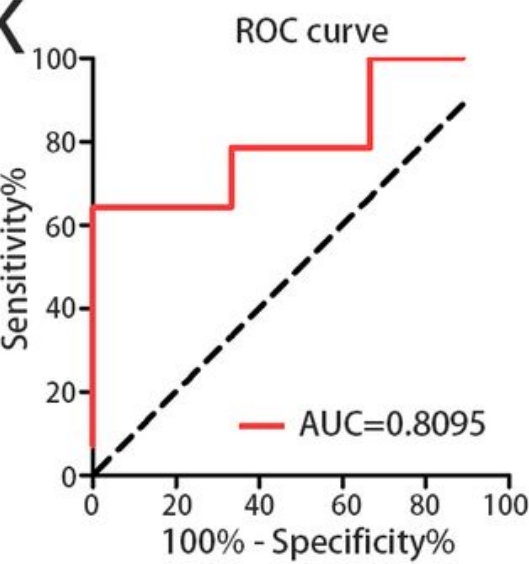

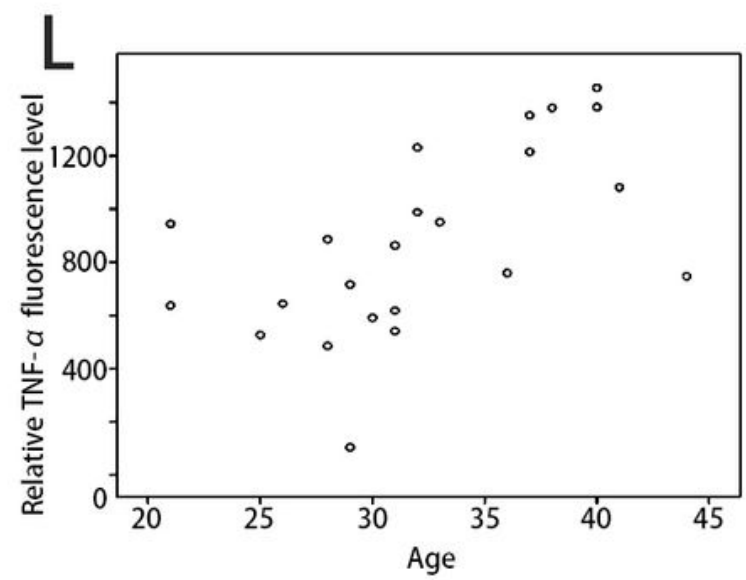

Figure 1

(A-J)10 cytokines in human embryo culture medium from IVF center were tested by the single cell cytokine array. ${ }^{\star \star} P<0.01$. Not all samples had signals at the time of testing. $(K)$ The ROC curve of TNF-a. (L) Correlation analysis of age and TNF-a expression in culture medium. 
A

IL- $1 \beta$
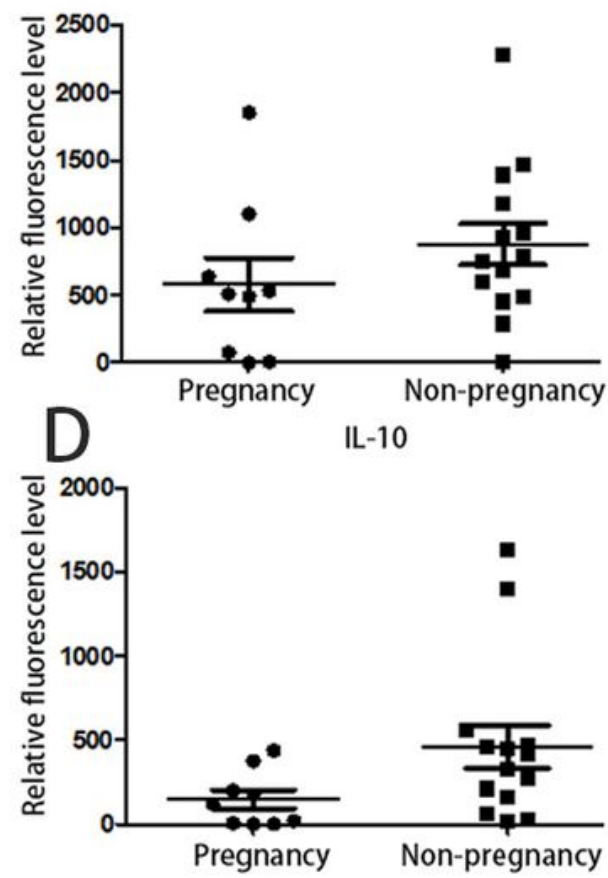

$G$
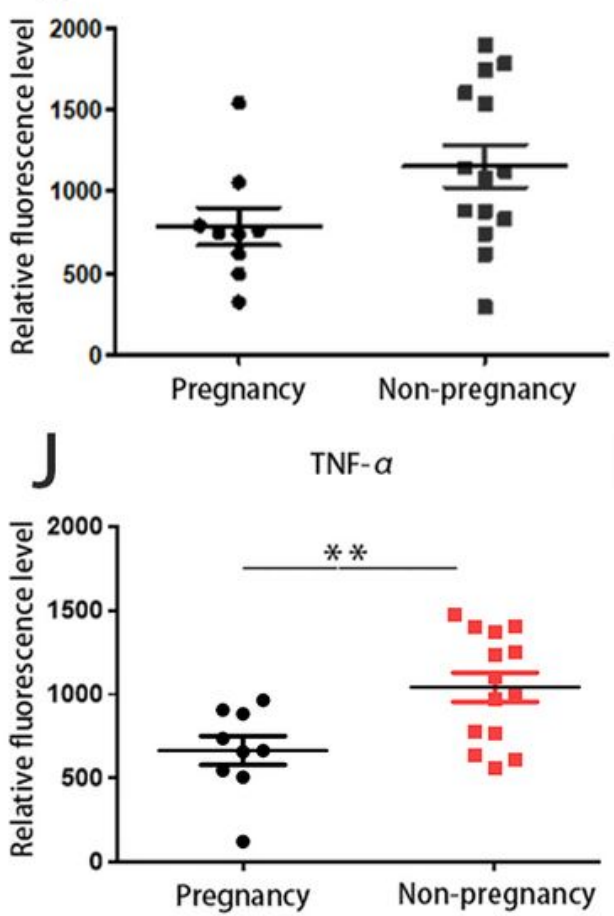

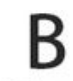

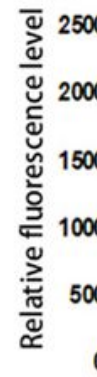

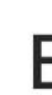

E
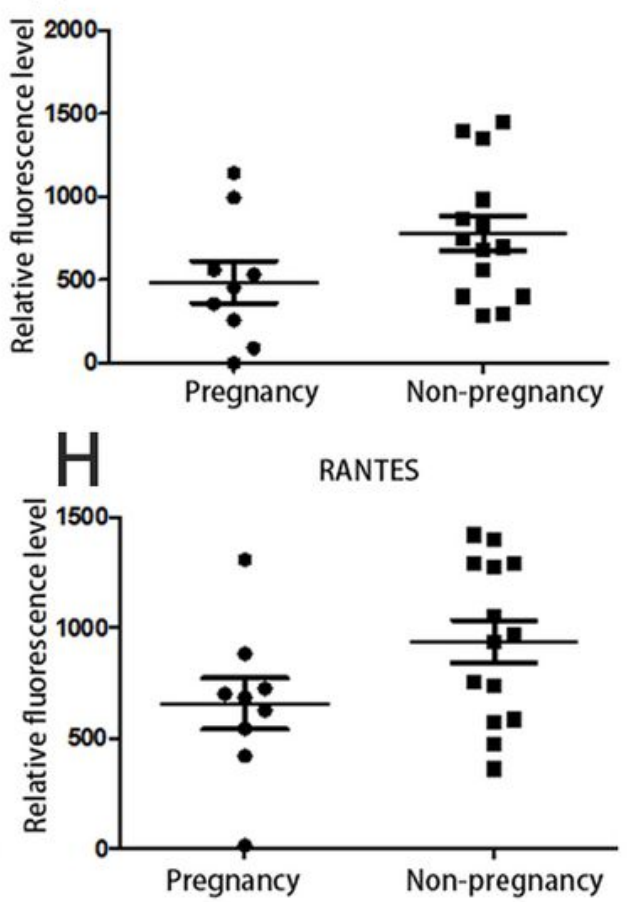

C

IL-8
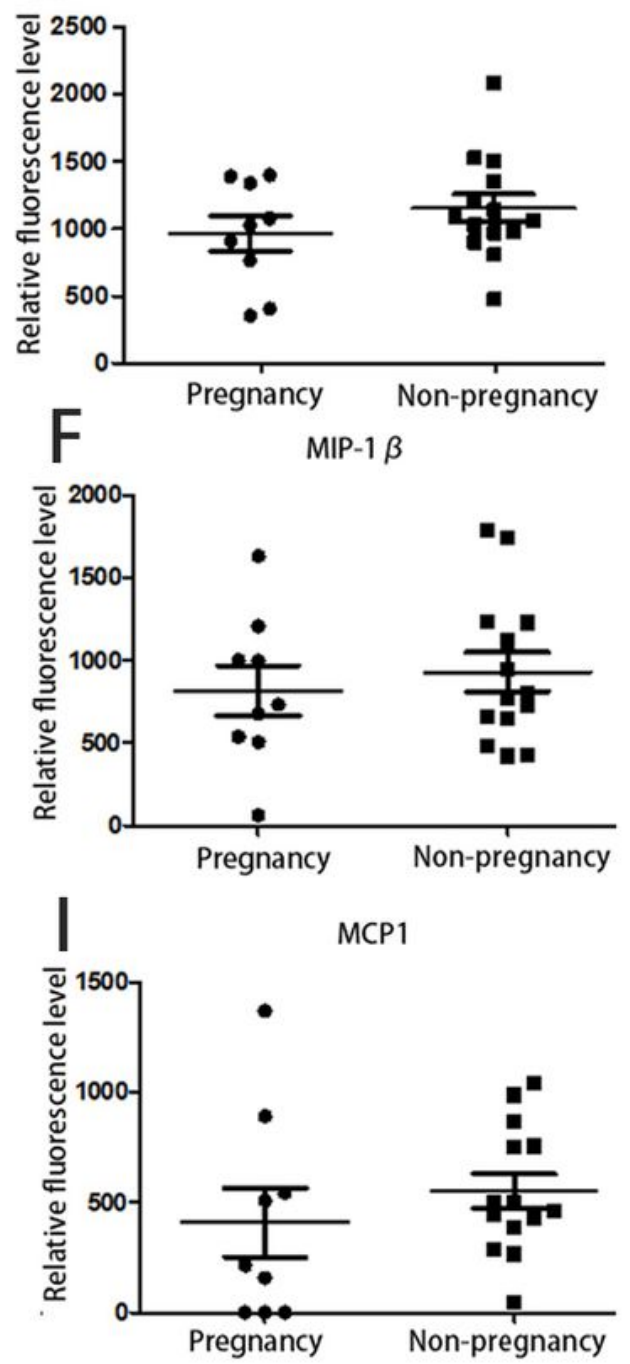

$\mathrm{K}_{100}$

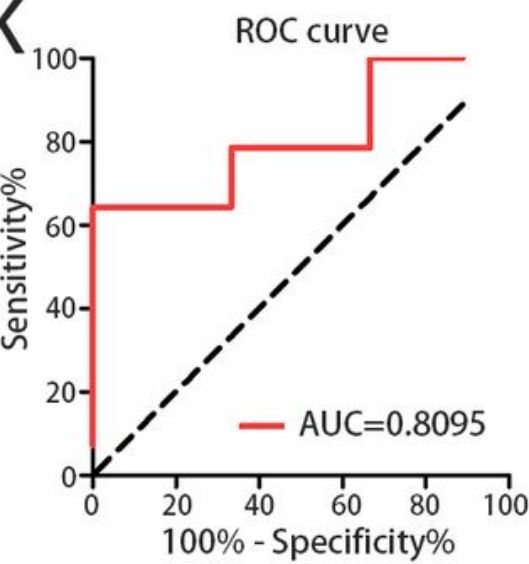

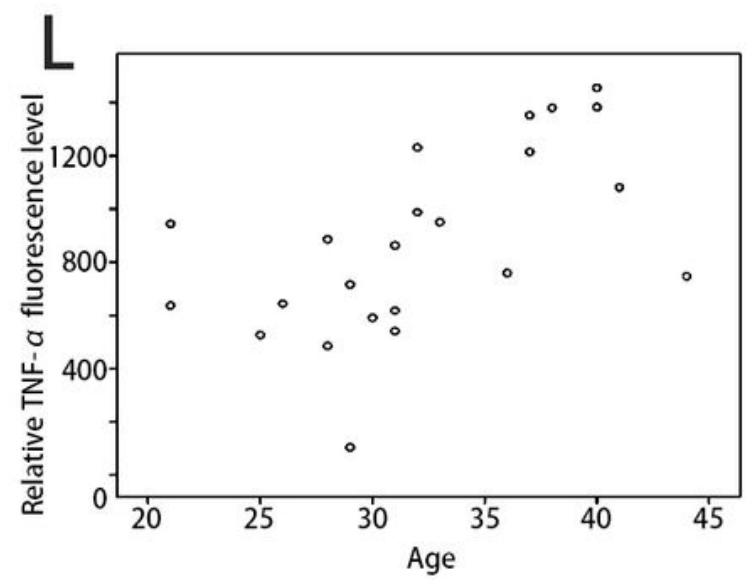

Figure 1

(A-J)10 cytokines in human embryo culture medium from IVF center were tested by the single cell cytokine array. ${ }^{\star \star} P<0.01$. Not all samples had signals at the time of testing. $(K)$ The ROC curve of TNF-a. (L) Correlation analysis of age and TNF-a expression in culture medium. 


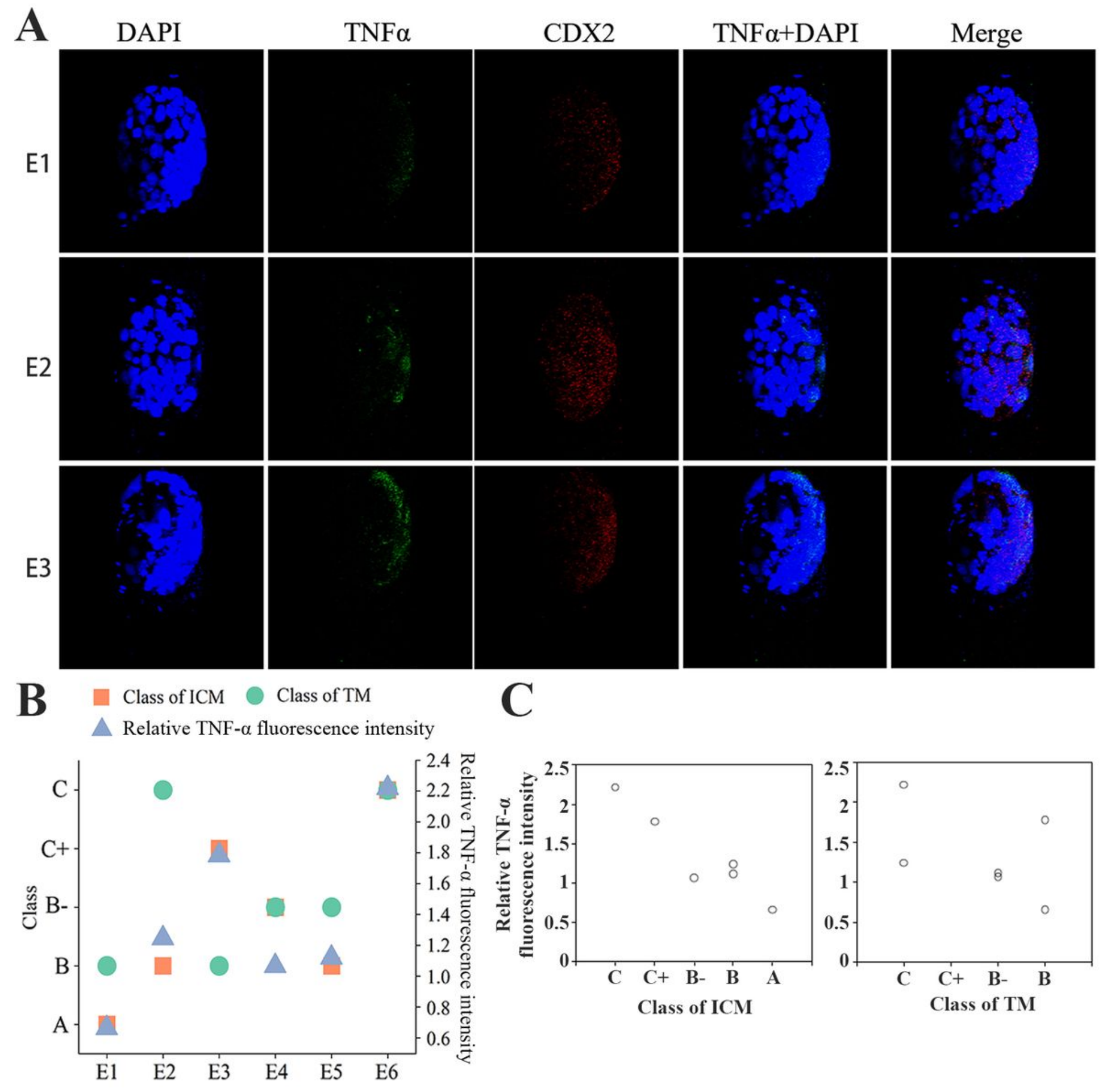

Figure 2

(A) The immunostaining of TNF-a (green) and CDX2 (red) in human normal blastocyst-stage embryo, nuclei were labelled with 4',6-diamidino-2-phenylindole (DAPI) (blue). Six embryos (E1-E6) were tested, while three were in supplementary data files. Magnification, $x 200$. We also provided video animation in supplementary. (B) Scatter plot analysis of the relationship between embryo quality and the relative fluorescence intensity of TNF- $a$ (relative to DAPI). (C) Correlation analysis of TNF- $a$ fluorescence intensity and the quality of embryo ICM or TM. 


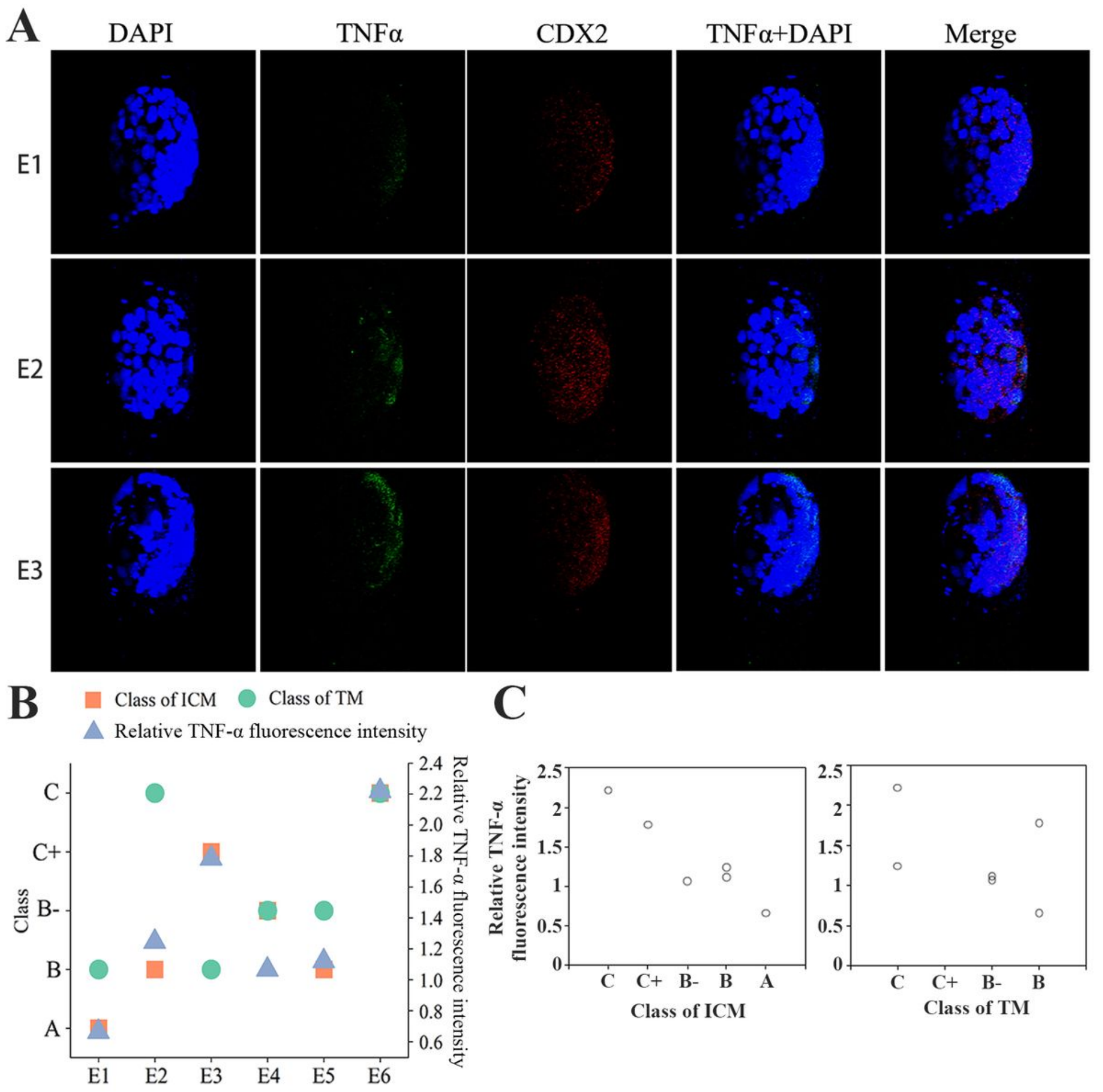

Figure 2

(A) The immunostaining of TNF-a (green) and CDX2 (red) in human normal blastocyst-stage embryo, nuclei were labelled with 4',6-diamidino-2-phenylindole (DAPI) (blue). Six embryos (E1-E6) were tested, while three were in supplementary data files. Magnification, $x 200$. We also provided video animation in supplementary. (B) Scatter plot analysis of the relationship between embryo quality and the relative fluorescence intensity of TNF- $a$ (relative to DAPI). (C) Correlation analysis of TNF-a fluorescence intensity and the quality of embryo ICM or TM. 


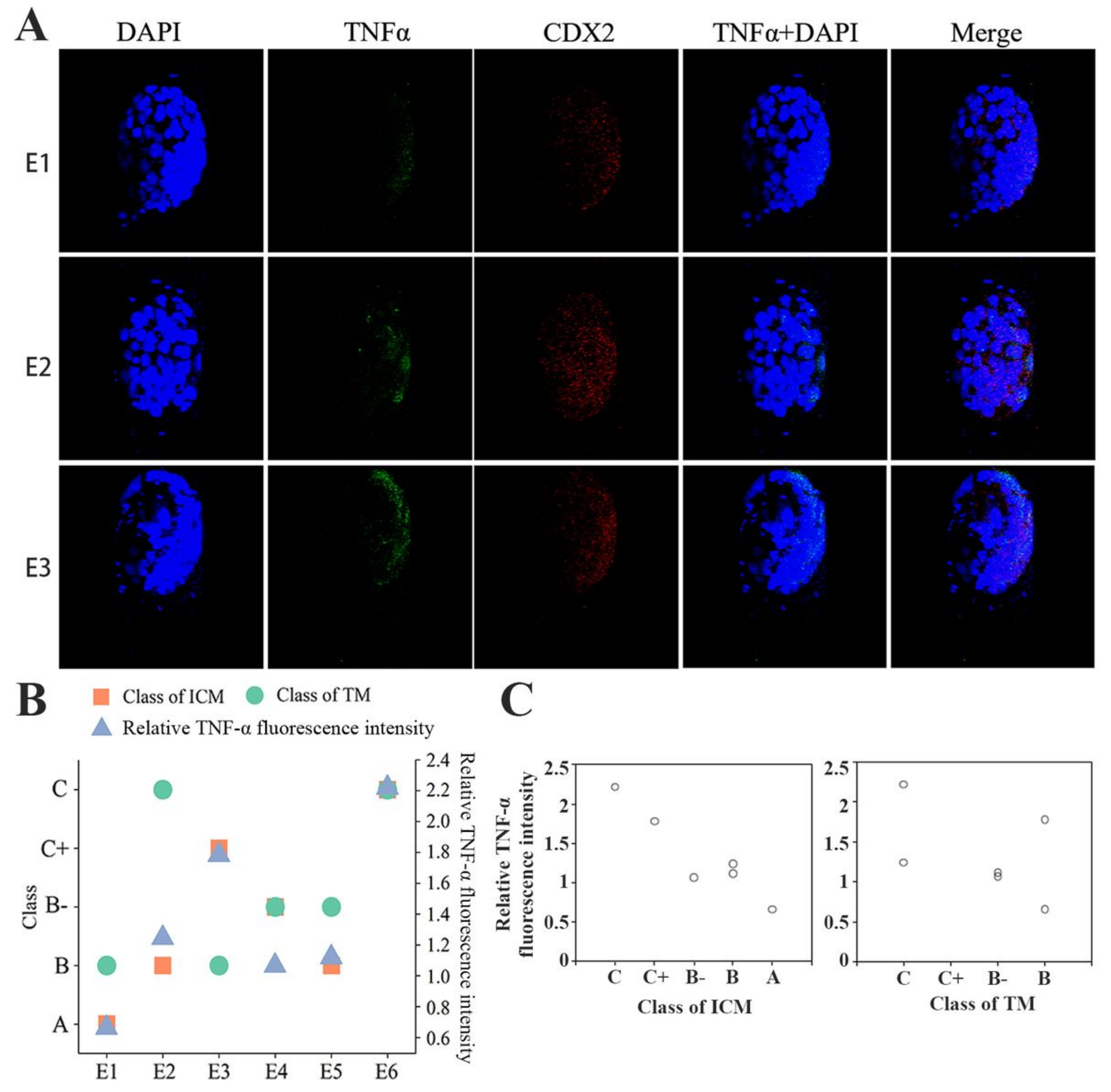

Figure 2

(A) The immunostaining of TNF-a (green) and CDX2 (red) in human normal blastocyst-stage embryo, nuclei were labelled with 4',6-diamidino-2-phenylindole (DAPI) (blue). Six embryos (E1-E6) were tested, while three were in supplementary data files. Magnification, $x 200$. We also provided video animation in supplementary. (B) Scatter plot analysis of the relationship between embryo quality and the relative fluorescence intensity of TNF- $a$ (relative to DAPI). (C) Correlation analysis of TNF- $a$ fluorescence intensity and the quality of embryo ICM or TM. 


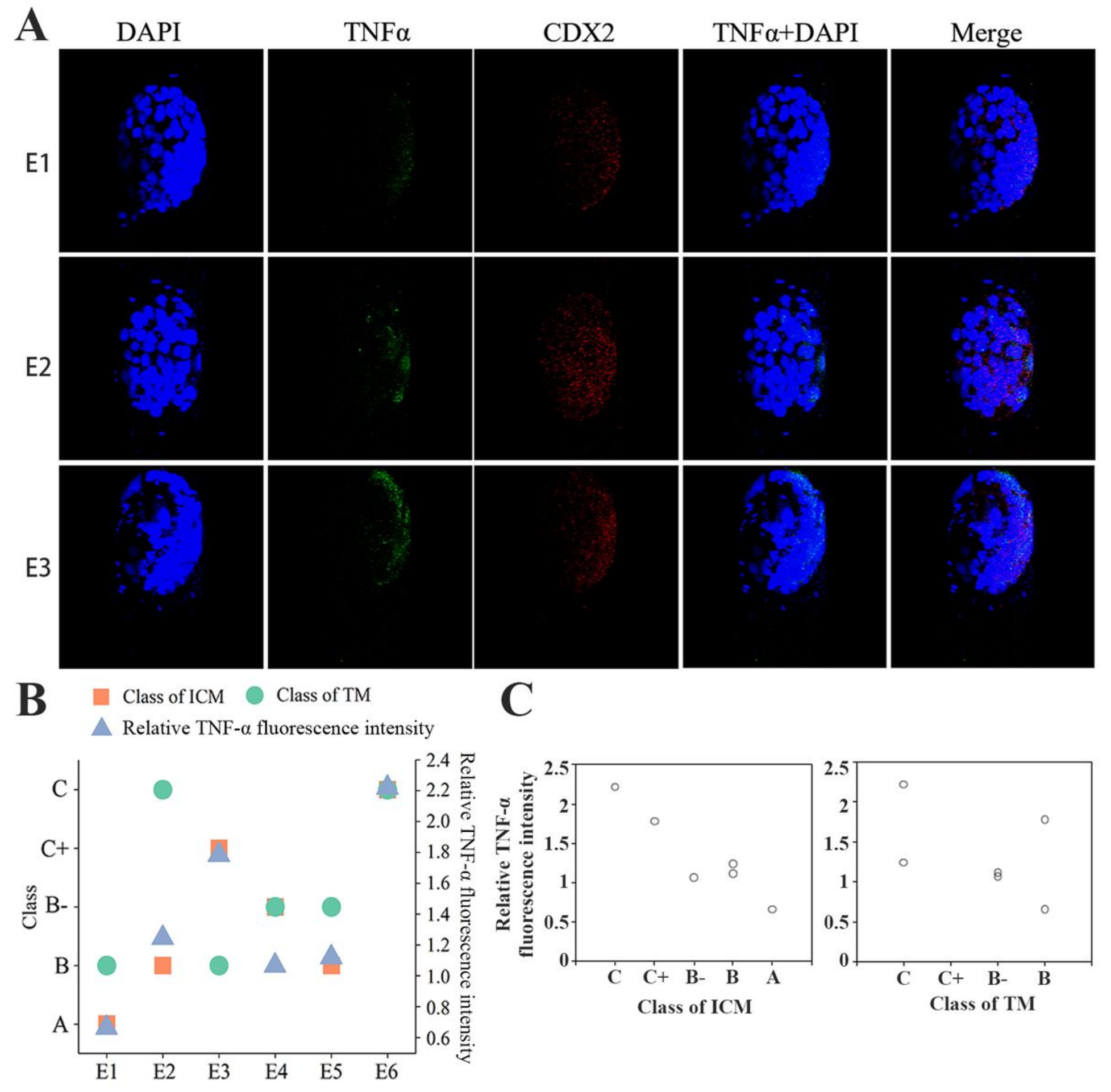

Figure 2

(A) The immunostaining of TNF-a (green) and CDX2 (red) in human normal blastocyst-stage embryo, nuclei were labelled with 4',6-diamidino-2-phenylindole (DAPI) (blue). Six embryos (E1-E6) were tested, while three were in supplementary data files. Magnification, $x 200$. We also provided video animation in supplementary. (B) Scatter plot analysis of the relationship between embryo quality and the relative fluorescence intensity of TNF- $a$ (relative to DAPI). (C) Correlation analysis of TNF- $a$ fluorescence intensity and the quality of embryo ICM or TM. 


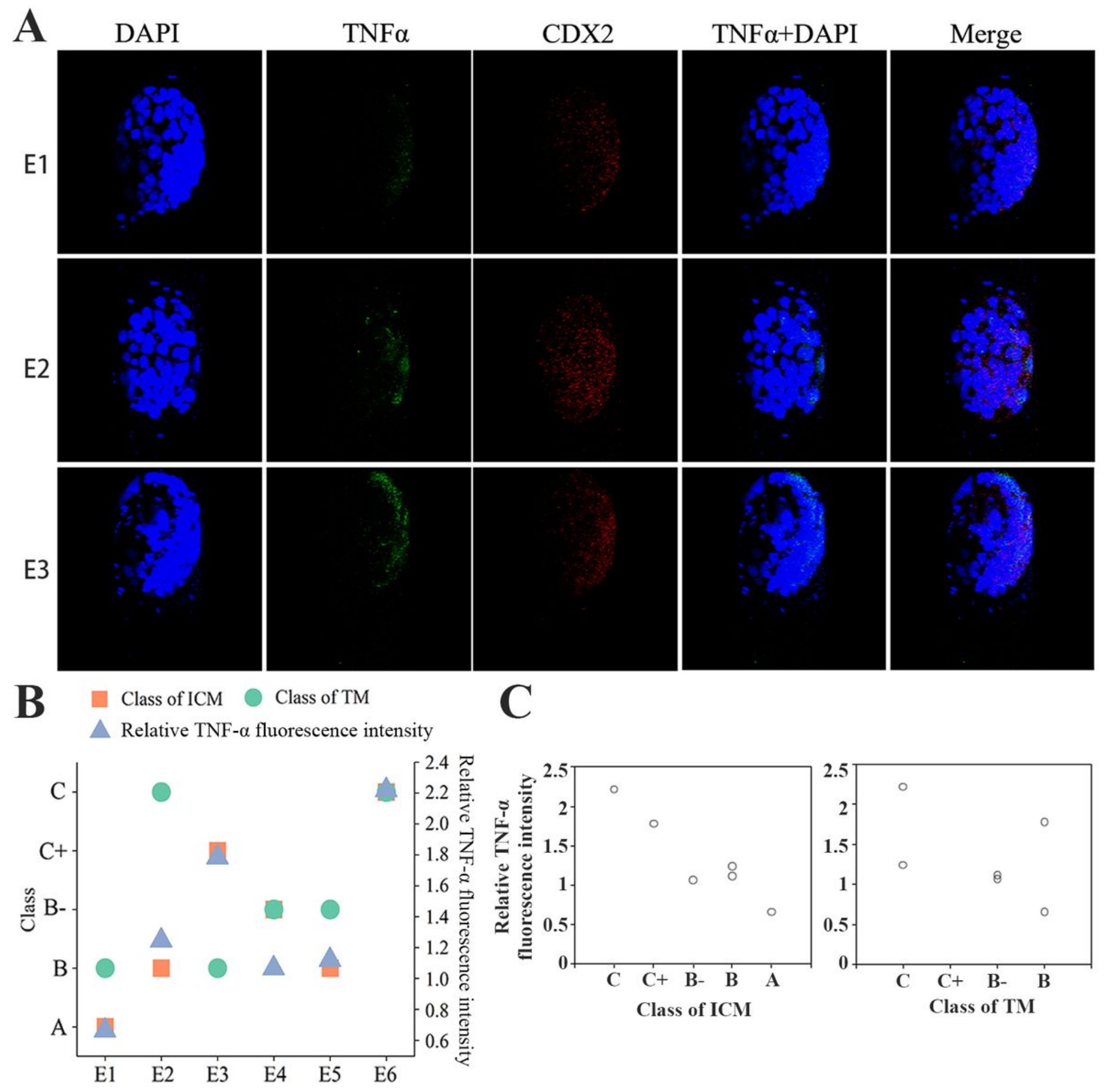

Figure 2

(A) The immunostaining of TNF-a (green) and CDX2 (red) in human normal blastocyst-stage embryo, nuclei were labelled with 4',6-diamidino-2-phenylindole (DAPI) (blue). Six embryos (E1-E6) were tested, while three were in supplementary data files. Magnification, $x 200$. We also provided video animation in supplementary. (B) Scatter plot analysis of the relationship between embryo quality and the relative fluorescence intensity of TNF- $a$ (relative to DAPI). (C) Correlation analysis of TNF-a fluorescence intensity and the quality of embryo ICM or TM. 


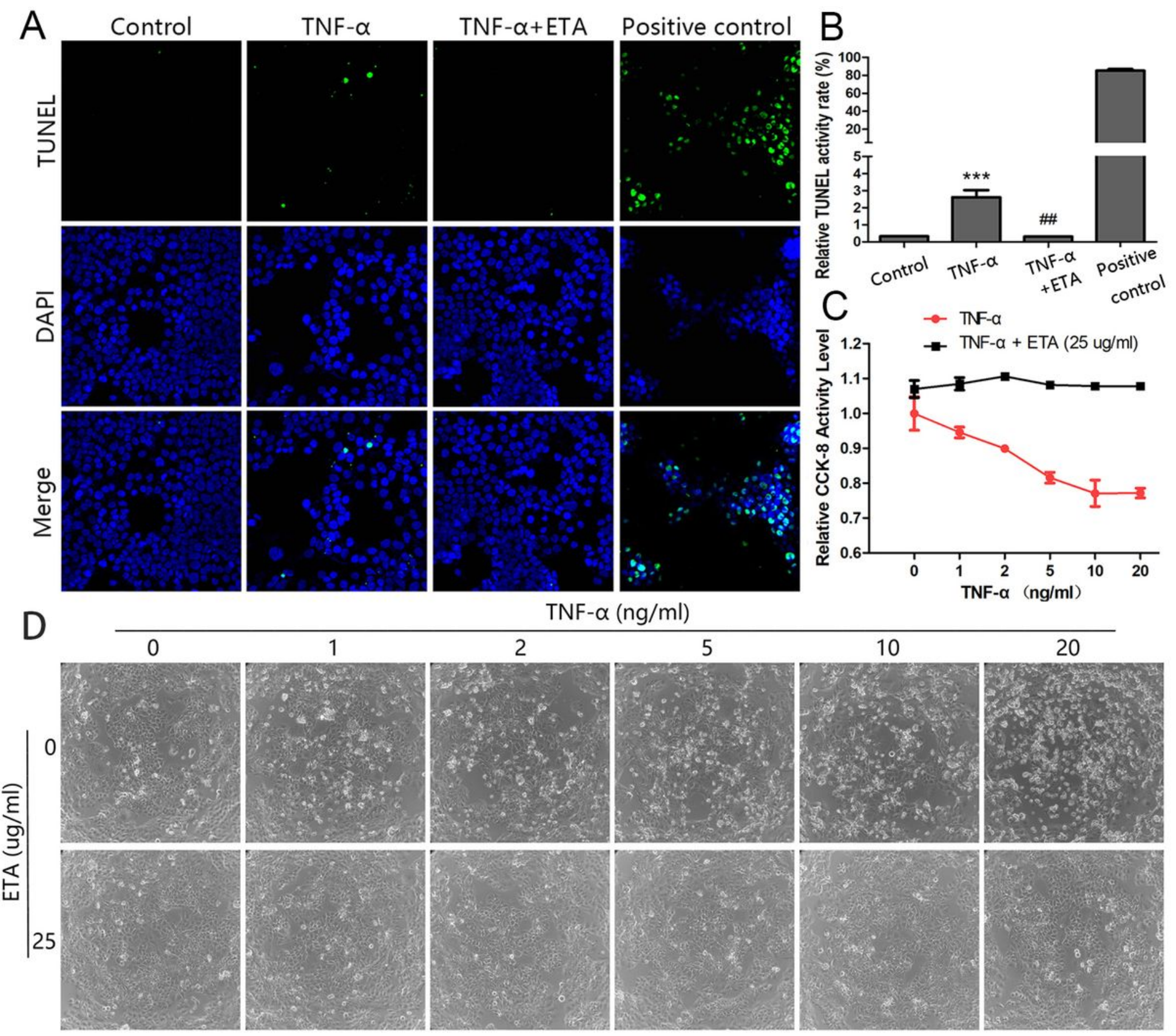

Figure 3

(A, B) TUNEL staining assay measuring cell apoptosis after $48 \mathrm{~h}$ incubation. $10 \mathrm{ng} / \mathrm{ml}$ TNF-a and 25 $\mu \mathrm{g} / \mathrm{ml}$ ETA were used in the test. Green, TUNEL stained nuclei of apoptosis cells; blue, all nuclei counterstained with DAPI. Magnification, $x 200 .{ }^{*} * *<<0.001$ vs. the control group, \#\#P<0.01 vs. the TNF-a group. (C, D) Add TNF-a lead to dose dependent inhibit cell proliferation in the Ishikawa cell after $48 \mathrm{~h}$ incubation, while adding ETA can recover the inhibition caused by TNF-a in the Ishikawa cell model, tested by CCK-8 assay. Each group was compared with the TNF-a $(0 \mathrm{ng} / \mathrm{ml})$ ETA $(0 \mu \mathrm{g} / \mathrm{ml})$ group, and that group was regarded as "1" for data statistics. Magnification, x200. 


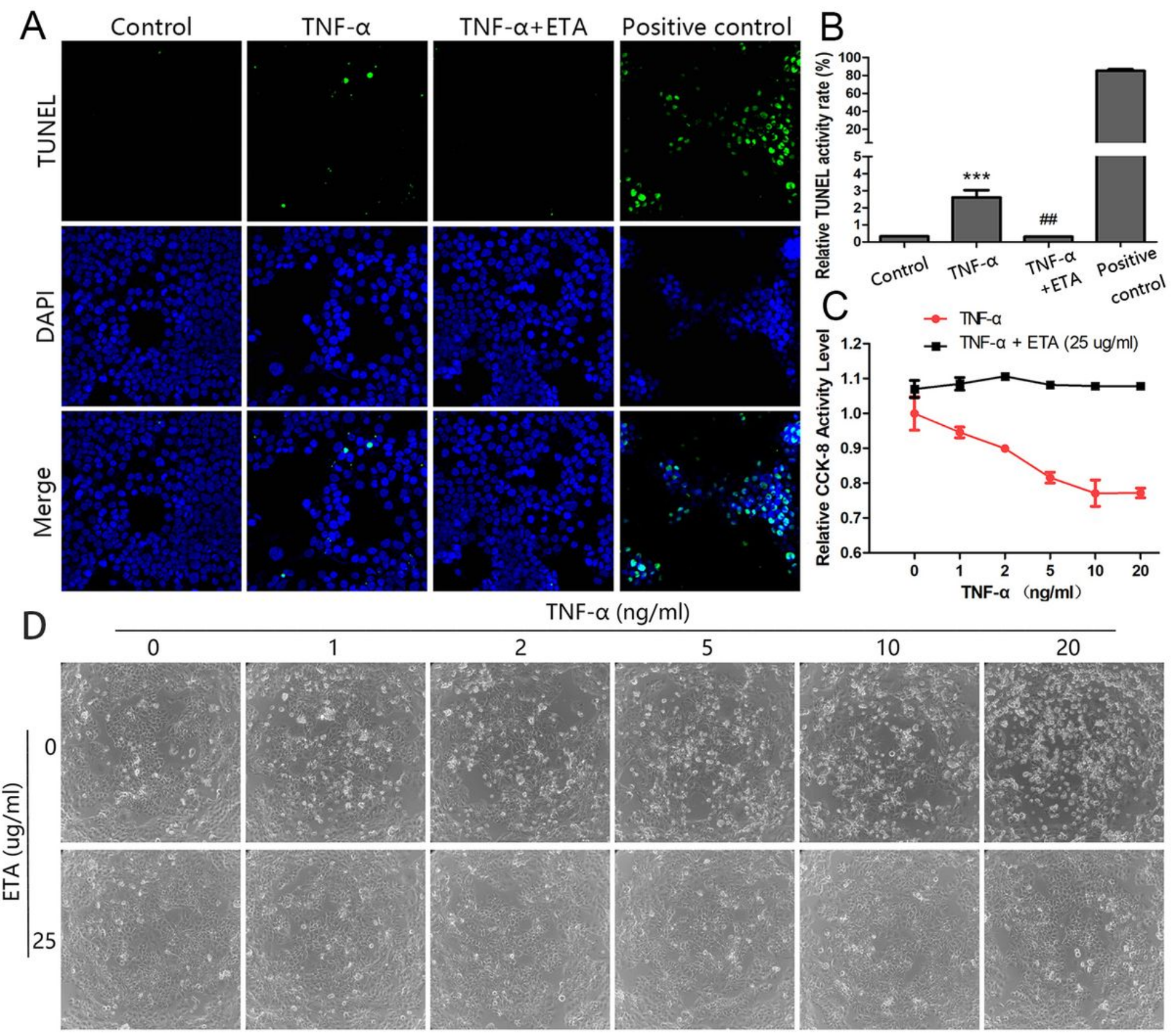

Figure 3

(A, B) TUNEL staining assay measuring cell apoptosis after $48 \mathrm{~h}$ incubation. $10 \mathrm{ng} / \mathrm{ml}$ TNF-a and 25 $\mu \mathrm{g} / \mathrm{ml}$ ETA were used in the test. Green, TUNEL stained nuclei of apoptosis cells; blue, all nuclei counterstained with DAPI. Magnification, $x 200 .{ }^{*} * *<<0.001$ vs. the control group, \#\#P<0.01 vs. the TNF-a group. (C, D) Add TNF-a lead to dose dependent inhibit cell proliferation in the Ishikawa cell after $48 \mathrm{~h}$ incubation, while adding ETA can recover the inhibition caused by TNF-a in the Ishikawa cell model, tested by CCK-8 assay. Each group was compared with the TNF-a $(0 \mathrm{ng} / \mathrm{ml})$ ETA $(0 \mu \mathrm{g} / \mathrm{ml})$ group, and that group was regarded as "1" for data statistics. Magnification, x200. 


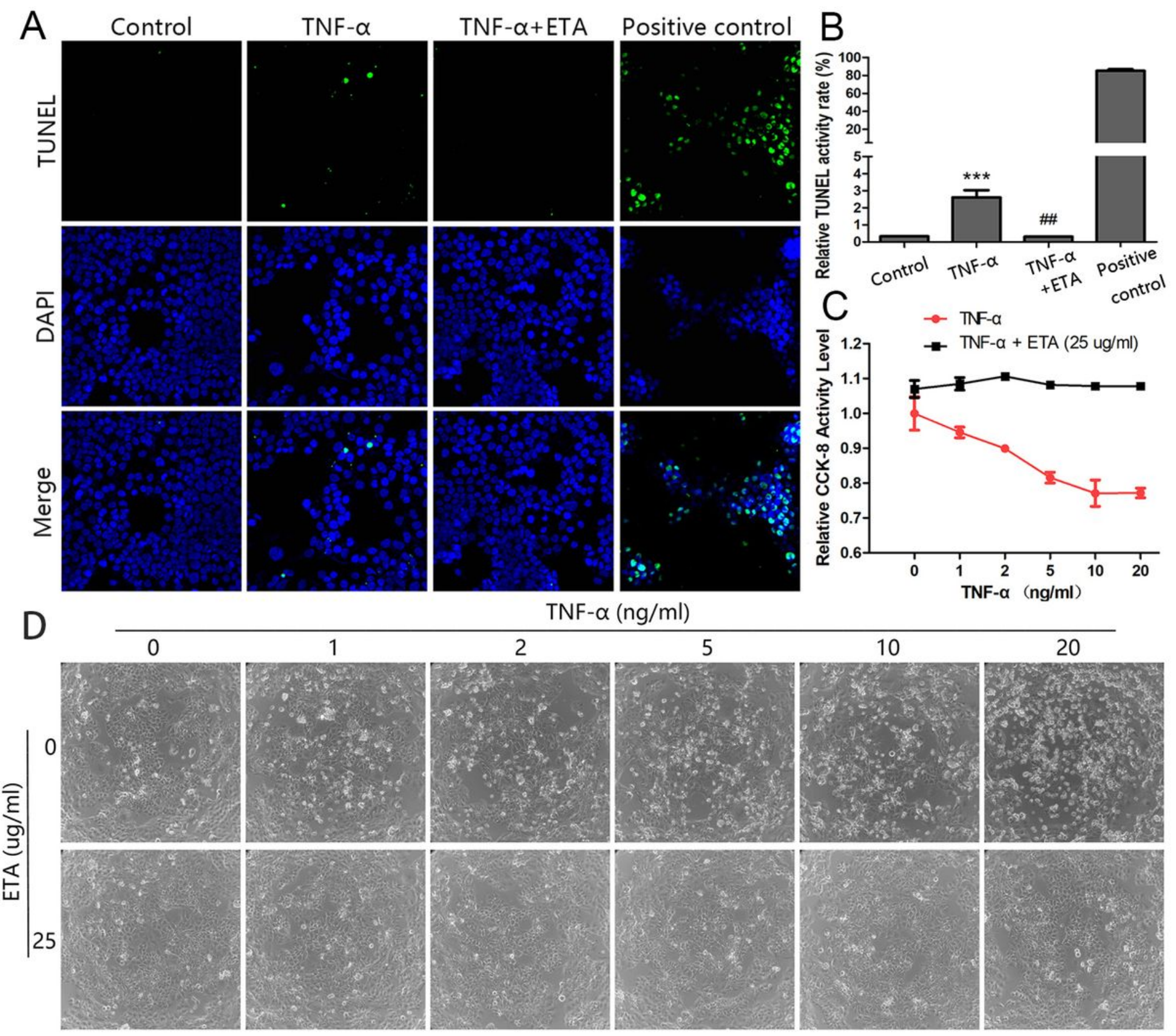

Figure 3

(A, B) TUNEL staining assay measuring cell apoptosis after $48 \mathrm{~h}$ incubation. $10 \mathrm{ng} / \mathrm{ml}$ TNF-a and 25 $\mu \mathrm{g} / \mathrm{ml}$ ETA were used in the test. Green, TUNEL stained nuclei of apoptosis cells; blue, all nuclei counterstained with DAPI. Magnification, $x 200 .{ }^{*} * *<<0.001$ vs. the control group, \#\#P<0.01 vs. the TNF-a group. (C, D) Add TNF-a lead to dose dependent inhibit cell proliferation in the Ishikawa cell after $48 \mathrm{~h}$ incubation, while adding ETA can recover the inhibition caused by TNF-a in the Ishikawa cell model, tested by CCK-8 assay. Each group was compared with the TNF-a $(0 \mathrm{ng} / \mathrm{ml})$ ETA $(0 \mu \mathrm{g} / \mathrm{ml})$ group, and that group was regarded as "1" for data statistics. Magnification, x200. 


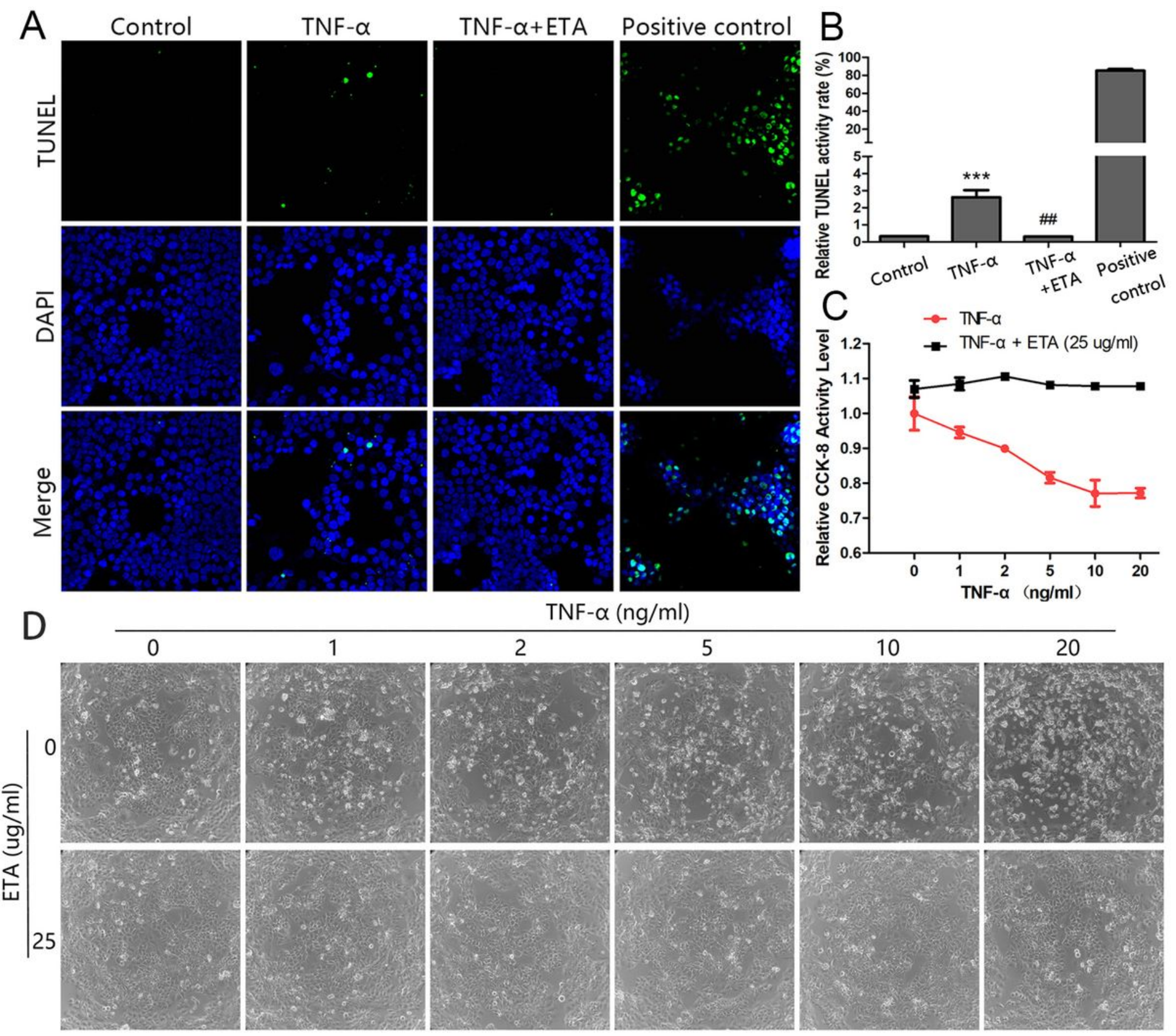

Figure 3

(A, B) TUNEL staining assay measuring cell apoptosis after $48 \mathrm{~h}$ incubation. $10 \mathrm{ng} / \mathrm{ml}$ TNF-a and 25 $\mu \mathrm{g} / \mathrm{ml}$ ETA were used in the test. Green, TUNEL stained nuclei of apoptosis cells; blue, all nuclei counterstained with DAPI. Magnification, $x 200 .{ }^{*} * *<<0.001$ vs. the control group, \#\#P<0.01 vs. the TNF-a group. (C, D) Add TNF-a lead to dose dependent inhibit cell proliferation in the Ishikawa cell after $48 \mathrm{~h}$ incubation, while adding ETA can recover the inhibition caused by TNF-a in the Ishikawa cell model, tested by CCK-8 assay. Each group was compared with the TNF-a $(0 \mathrm{ng} / \mathrm{ml})$ ETA $(0 \mu \mathrm{g} / \mathrm{ml})$ group, and that group was regarded as "1" for data statistics. Magnification, x200. 


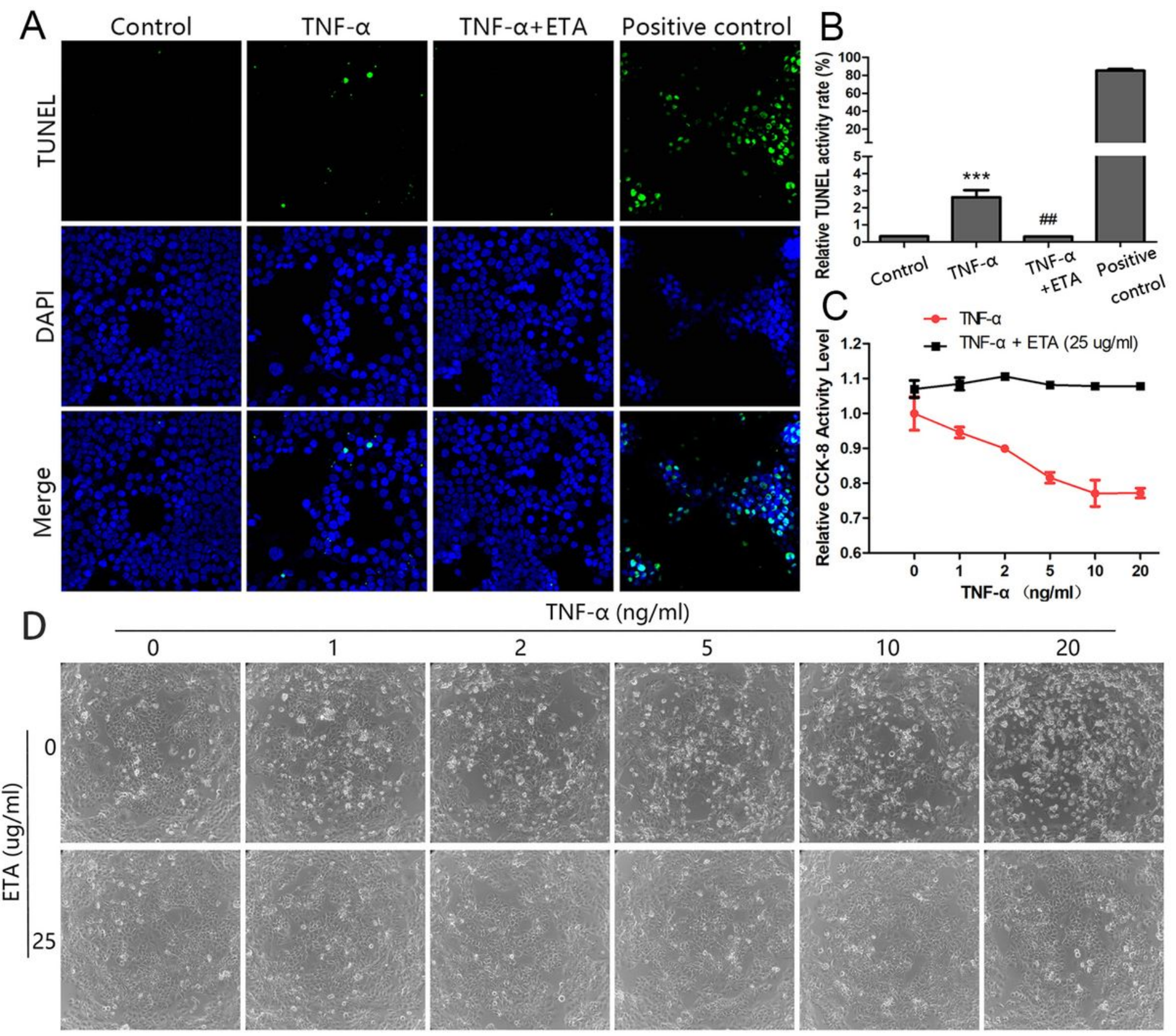

Figure 3

(A, B) TUNEL staining assay measuring cell apoptosis after $48 \mathrm{~h}$ incubation. $10 \mathrm{ng} / \mathrm{ml}$ TNF-a and 25 $\mu \mathrm{g} / \mathrm{ml}$ ETA were used in the test. Green, TUNEL stained nuclei of apoptosis cells; blue, all nuclei counterstained with DAPI. Magnification, $x 200 .{ }^{*} * *<<0.001$ vs. the control group, \#\#P<0.01 vs. the TNF-a group. (C, D) Add TNF-a lead to dose dependent inhibit cell proliferation in the Ishikawa cell after $48 \mathrm{~h}$ incubation, while adding ETA can recover the inhibition caused by TNF-a in the Ishikawa cell model, tested by CCK-8 assay. Each group was compared with the TNF-a $(0 \mathrm{ng} / \mathrm{ml})$ ETA $(0 \mu \mathrm{g} / \mathrm{ml})$ group, and that group was regarded as "1" for data statistics. Magnification, x200. 

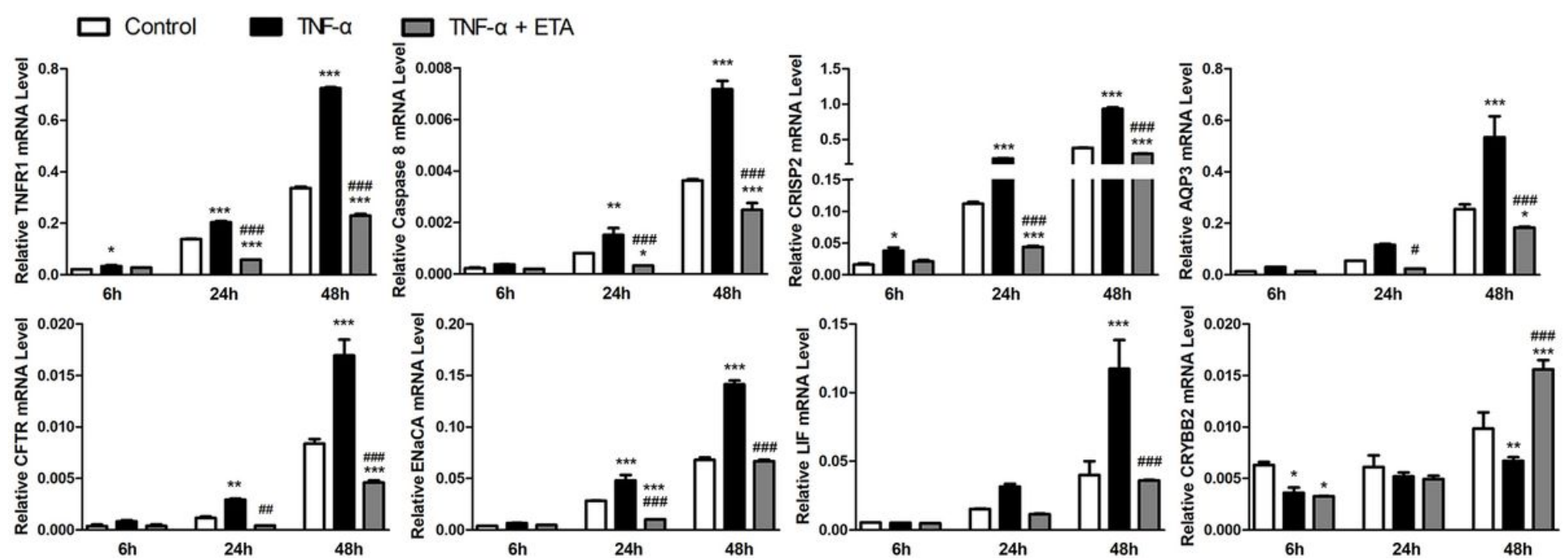

Figure 4

Adding $10 \mathrm{ng} / \mathrm{ml}$ TNF-a can decrease the expression of CRYBB2 and increase of TNFR1, Caspase8, CRISP2, AQP3, CFTR, ENaCA and LIF in the Ishikawa cell line after 48h incubation, while pre-treatment of $25 \mu \mathrm{g} / \mathrm{ml}$ ETA can significantly inhibit the action of TNF-a. ${ }^{*}<<0.05$ and ${ }^{\star *} * \mathrm{P}<0.001$ vs. the control group, \#\#\#P<0.001 vs. the TNF-a group.
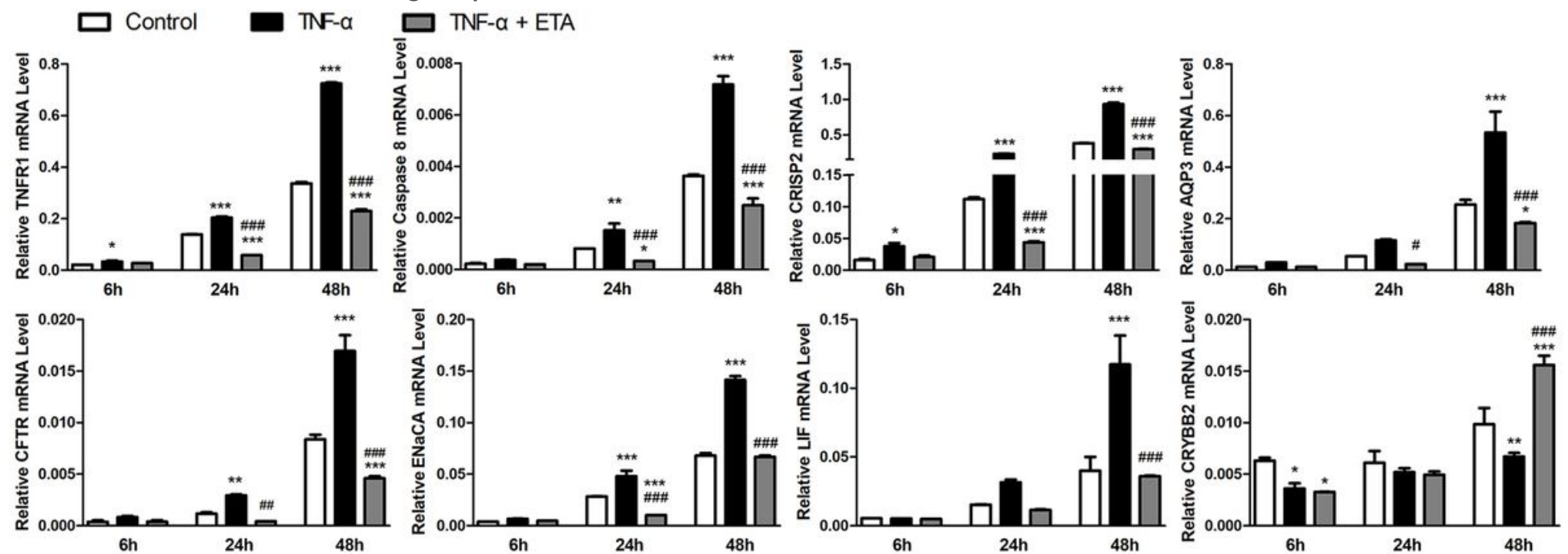

Figure 4

Adding $10 \mathrm{ng} / \mathrm{ml}$ TNF-a can decrease the expression of CRYBB2 and increase of TNFR1, Caspase8, CRISP2, AQP3, CFTR, ENaCA and LIF in the Ishikawa cell line after 48h incubation, while pre-treatment of $25 \mu \mathrm{g} / \mathrm{ml}$ ETA can significantly inhibit the action of TNF- $a$. ${ }^{*}<<0.05$ and ${ }^{*} * *<<0.001$ vs. the control group, \#\#\#P<0.001 vs. the TNF-a group. 

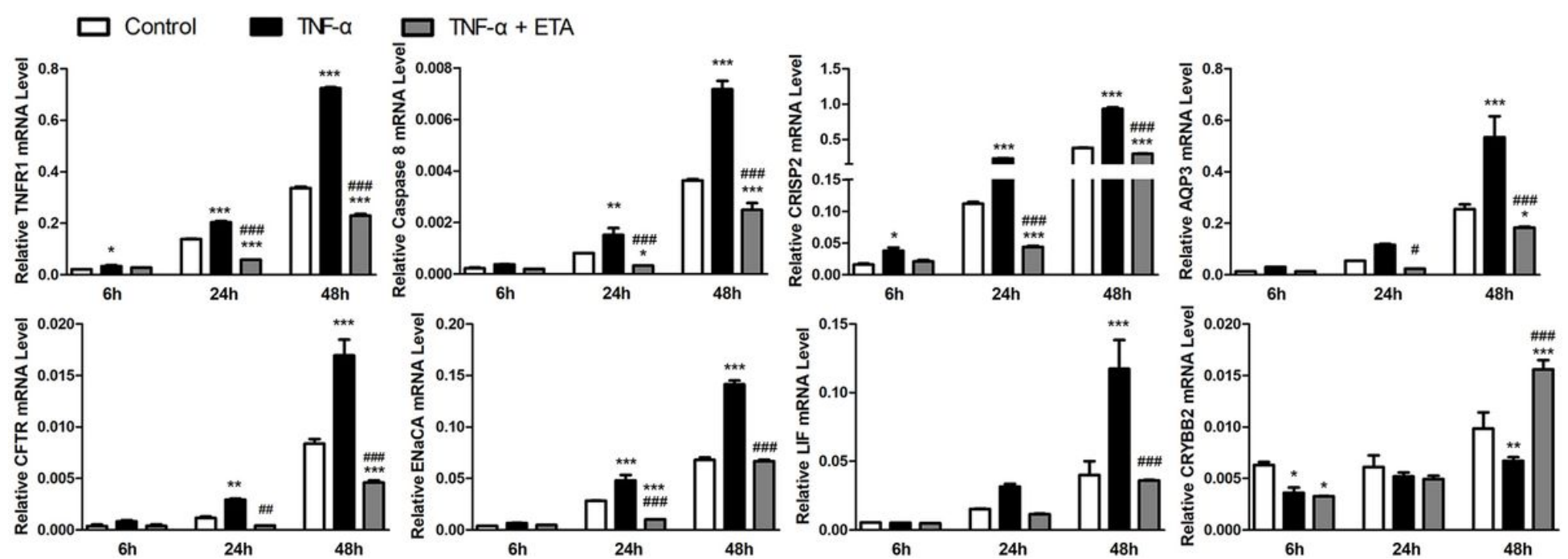

Figure 4

Adding $10 \mathrm{ng} / \mathrm{ml}$ TNF-a can decrease the expression of CRYBB2 and increase of TNFR1, Caspase8, CRISP2, AQP3, CFTR, ENaCA and LIF in the Ishikawa cell line after 48h incubation, while pre-treatment of $25 \mu \mathrm{g} / \mathrm{ml}$ ETA can significantly inhibit the action of TNF-a. ${ }^{*}<<0.05$ and ${ }^{\star *} * \mathrm{P}<0.001$ vs. the control group, \#\#\#P<0.001 vs. the TNF-a group.
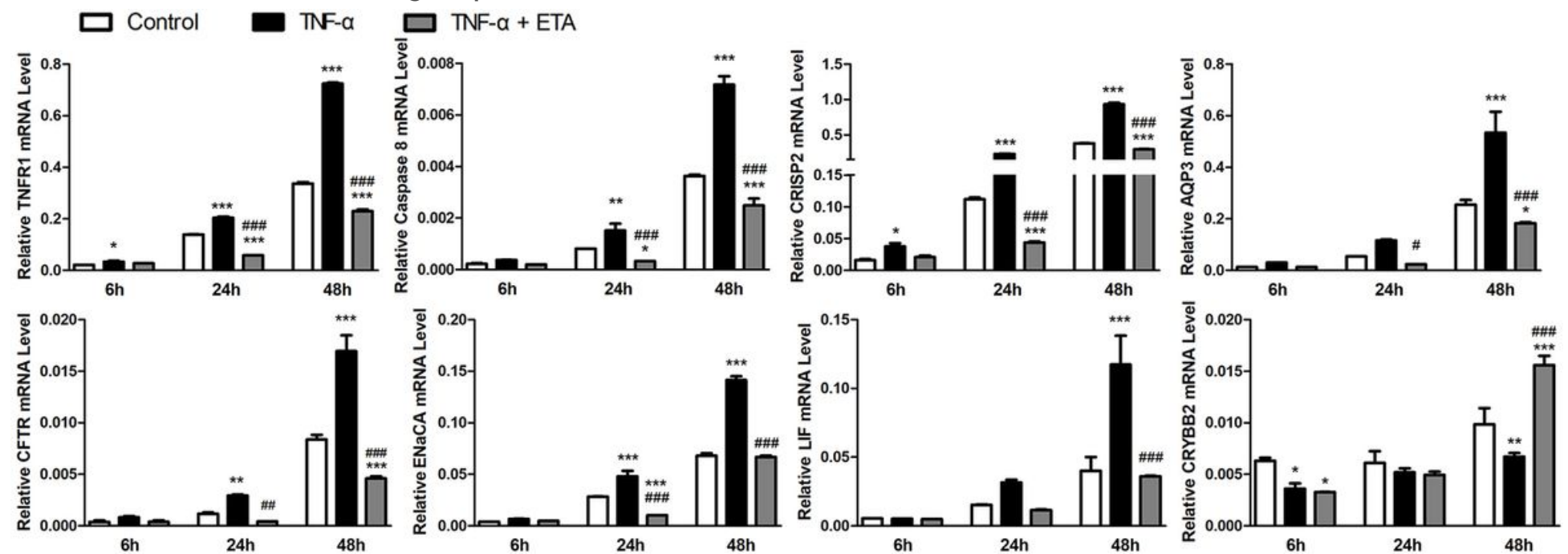

Figure 4

Adding $10 \mathrm{ng} / \mathrm{ml}$ TNF-a can decrease the expression of CRYBB2 and increase of TNFR1, Caspase8, CRISP2, AQP3, CFTR, ENaCA and LIF in the Ishikawa cell line after 48h incubation, while pre-treatment of $25 \mu \mathrm{g} / \mathrm{ml}$ ETA can significantly inhibit the action of TNF- $a$. ${ }^{*}<<0.05$ and ${ }^{*} * *<<0.001$ vs. the control group, \#\#\#P<0.001 vs. the TNF-a group. 

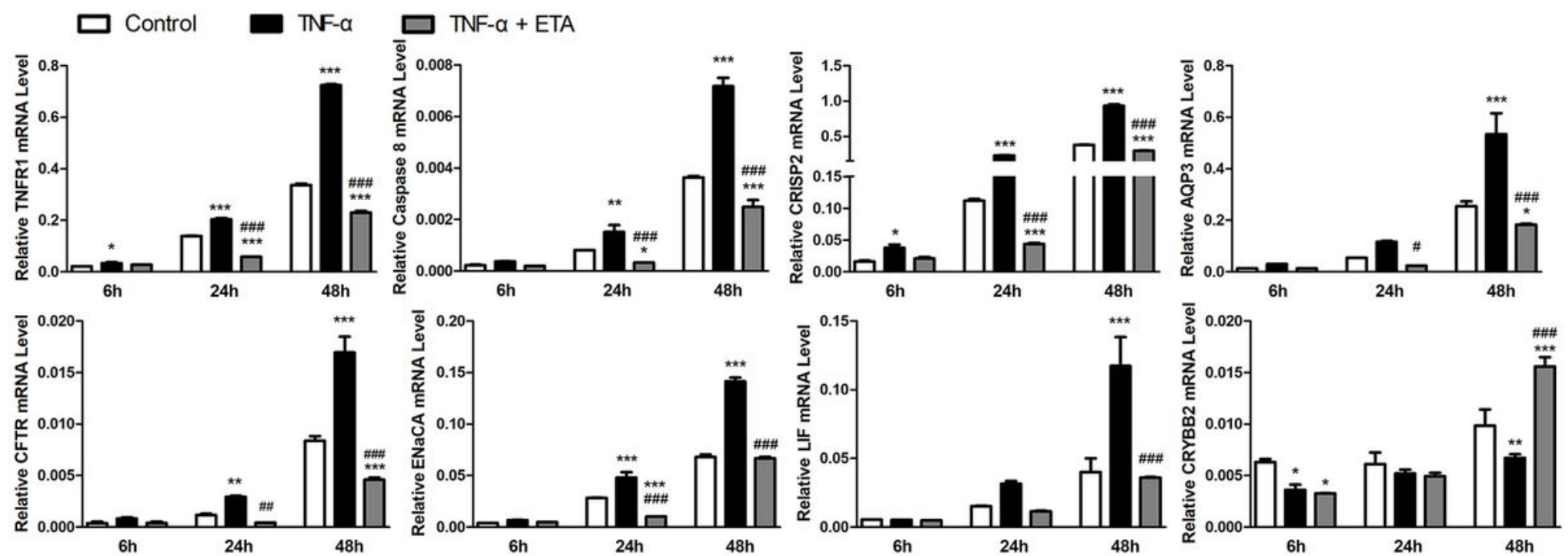

Figure 4

Adding $10 \mathrm{ng} / \mathrm{ml}$ TNF-a can decrease the expression of CRYBB2 and increase of TNFR1, Caspase8, CRISP2, AQP3, CFTR, ENaCA and LIF in the Ishikawa cell line after 48h incubation, while pre-treatment of $25 \mu \mathrm{g} / \mathrm{ml}$ ETA can significantly inhibit the action of TNF-a. ${ }^{*}<<0.05$ and ${ }^{\star *} * \mathrm{P}<0.001$ vs. the control group, \#\#\#P<0.001 vs. the TNF-a group.

\section{Supplementary Files}

This is a list of supplementary files associated with this preprint. Click to download.

- E1Movie.mp4

- E1Movie.mp4

- E1Movie.mp4

- E1Movie.mp4

- E1Movie.mp4

- E2Movie.mp4

- E2Movie.mp4

- E2Movie.mp4

- E2Movie.mp4

- E2Movie.mp4

- E3Movie.mp4

- E3Movie.mp4

- E3Movie.mp4

- E3Movie.mp4

- E3Movie.mp4 
- E4Movie.mp4

- E4Movie.mp4

- E4Movie.mp4

- E4Movie.mp4

- E4Movie.mp4

- E5Movie.mp4

- E5Movie.mp4

- E5Movie.mp4

- E5Movie.mp4

- E5Movie.mp4

- E6Movie.mp4

- E6Movie.mp4

- E6Movie.mp4

- E6Movie.mp4

- E6Movie.mp4

- supplementaryfigure1.jpg

- supplementaryfigure1.jpg

- supplementaryfigure1.jpg

- supplementaryfigure1.jpg

- supplementaryfigure1.jpg 